\title{
New, Like New, or Very Good? Reputation and Credibility
}

\author{
BRUNO JULLIEN \\ Toulouse School of Economics \\ CNRS, GREMAQ and IDEI
}

\author{
IN-UCK PARK \\ University of Bristol \\ Sungkyunkwan University
}

January 29, 2014

\begin{abstract}
We show that sellers may earn reputation for their "ability" to deliver high quality goods on average by honestly announcing the realised quality of items for sale every period. As the expected revenue stream from continuing with honest communication increases with their ability, high ability sellers remain honest while low ability sellers find it too costly and sometimes lie about quality for short-term gain. Thus, cheap-talk communication facilitates the market's learning of a seller's ability and strengthens reputation effects. We study this new reputation mechanism and the induced market dynamics, first when sellers cannot restart with a new identity and then when they can. We extend the analysis to various other situations such as voluntary refund and moral hazard. (JEL Codes: C73, D82, D83, L14)
\end{abstract}

Keywords: communication, consumer rating system, credibility, reputation.

\section{Introduction}

Consider a seller who supplies an experience good repeatedly, with varying quality. Suppose that the seller can communicate about product quality and that potential buyers have access to the experience of past buyers with this seller to evaluate his claim. How much confidence should buyers have that the seller communicates the quality truthfully? As an example, a prospective buyer of a used iPhone on an Internet platform such as Amazon Marketplace may find two advertisements: one seller claims the phone to be in "Very Good" condition while another seller claims the phone to be "Like New", both asking for similar prices. The buyer would then look at the "feedback scores" of the two sellers on the platform that summarise the ratings by previous buyers, and also browse through the comments left by some of them, in order to judge how credible their claims are and thereby, which of the two phones to go for. Our objective is to understand the effects of such interactions when no formal consumer protection is available such as contractual warranty.

Notice that in such situations, if the seller trades only once, no effective communication on the product's quality may take place because the seller will always claim the best quality to get a higher price. Even if the seller trades repeatedly, the same is true if the average quality of the seller's product is commonly known, so long as the market pays for each item a price equal to its expected quality. The reason for this is that the expected revenue of the seller in each future period is equal to the known average quality, and is independent of what happens in the current period. 
In this article, we show that effective communication on product quality arises and facilitates trading if quality is random and there is uncertainty about the average quality of a seller's product, which we call the seller's "ability". In this environment, reputation represents the ability of a seller, but a seller's reputation is earned and maintained in the market by communicating the sale item's quality truthfully every time. As the expected revenue stream from such trustworthy behaviour reflects the seller's true ability, the reward from maintaining reputation is higher for high ability sellers. In equilibrium, the value differential is such that high ability sellers do not have any temptation to lie while low ability sellers sometimes lie about quality. Thus, the market's perception that ability comes with honesty is self-confirming. As a result, communication on product quality expedites buyers' learning of a seller's ability and the market lends more credibility to more reputable sellers.

This reputation mechanism applies to experience good markets generally, provided that the buyers can use the market's past experience with a particular seller to better assess that seller's claims. Specific examples include markets for services, cultural goods or wine, as well as Internet markets, where buyers share information about whether or not the sellers lived up to their word, through consumer reviews or direct word of mouth. ${ }^{1}$ Many internet markets (such as Amazon and eBay) have formal feedback systems in place for such information sharing. ${ }^{2}$

Our base model depicts a stylised environment where the quality of each item (which is freshly drawn every period) may be good or bad, and the seller may be of one of two private types, high or low ability: a high ability seller draws a good quality item with a higher probability. After a public cheap-talk announcement by the seller on the drawn quality, each period's item is traded at a price equal to the expected quality based on the information available in the market. After purchase, the buyer learns and publicly reports the true quality, revealing whether the seller was truthful or not. The market then updates its belief regarding the seller's ability, termed the seller's "reputation ", and the next period proceeds in the same manner.

As the seller's announcements are cheap talk, there always exists a "babbling" equilibrium in which the seller's announcements play no role and only the buyers' reports induce learning on the seller's ability through simple observation of past quality.

We focus on equilibria in which high ability sellers always announce truthfully, and we establish that there is a unique equilibrium of this kind, which we refer to as the "honest equilibrium". ${ }^{3}$ In this equilibrium, low ability sellers falsely claim bad quality items to be good with a positive probability and as a result, truthful announcements of bad quality increase the seller's reputation. The probability of a low ability seller lying is a continuous but non-monotonic function of the prevailing reputation level, reaching one above a certain

\footnotetext{
${ }^{1}$ See Bar-Isaac and Tadelis (2008) for a survey of the literature on seller reputation, and Dellarocas $(2003,2006)$ for a discussion of reputation issues on Internet markets.

${ }^{2}$ It is worth noting that accurate information from the seller is only one of the issues that feedback systems try to address. For recent evidence on the effect of the "feedback score", see Cabral and Hortascu (2010), Jolivet, Jullien, and Postel-Vinay (2013) and references therein. Jin and Kato (2006) provide evidence on eBay that communication is more reliable when the seller is reputable.

${ }^{3}$ We adopt this terminology from Sobel (1985).
} 
threshold reputation level.

Thus, communication facilitates information transmission along two dimensions in this equilibrium. Firstly, buyers are better informed about each item's quality. Secondly, buyers learn a seller's ability faster because announcements on quality transmit information on the seller's ability as well. A low ability seller lies less frequently as he becomes more patient, which exerts diverging effects on the two dimensions of information transmission. It enhances the credibility of the announcements on quality but it attenuates the speed at which the market learns the seller's ability.

We conduct the main analysis presuming that the seller observes the realised quality perfectly and that trade is always efficient, which helps separate the core insights from other effects but may raise issues of robustness and precludes welfare effects. To address these issues, we show that our main results continue to hold when the seller's observation of quality is subject to a small error. In addition, we extend the analysis in two directions that carry positive welfare implications: a moral hazard setting with differential costs of exerting effort and a setting where trading of a bad quality item is inefficient.

While our main analysis focuses on pre-trade communication, we show that the key insights are also applicable to situations where the sellers observe the realised quality only after trade. There, reputation concerns induce high ability sellers to voluntarily issue reimbursements for bad quality items sold, when this is observable by future consumers. ${ }^{4,5}$

Lastly, we study a situation in which sellers can start afresh at any time by obtaining a new identity after "milking" their reputation, which is a well-known issue in Internet markets. ${ }^{6}$ Allowing for a constant flow of entry and exit of sellers, we establish that honest equilibrim continues to exist in this environment. Compared with the baseline model, however, as restart options increase cheating incentives, low ability sellers lie more frequently. The price for a good quality item is thus lower for sellers of all reputation but high ability sellers build reputation faster. In addition, newcomers suffer from a depressed initial reputation because they are indistinguishable from the restarting, low ability sellers.

Our paper contributes to two strands of the reputation literature ${ }^{7}$ initiated by Kreps and Wilson (1982) and Milgrom and Roberts (1982), namely, the literature on sellers' reputation and that on the credibility of communication. By bridging these two strands of literature, we uncover a novel reputation mechanism with fresh insights.

In the former strand of literature, surveyed by Bar-Isaac and Tadelis (2008), reputation

\footnotetext{
${ }^{4}$ The idea that warranty may substitute for credible communication of quality dates back at least to Grossman (1981). Recently, Inderst and Ottaviani (2013) have pointed to a complementarity between warranty and pre-trade communication.

${ }^{5}$ There is some discretion in refund policy, in particular because sellers must evaluate good-faith and avoid fraudulent behaviors (see "Don't Even Think About Returning That Dress", BloombergBusinessWeek, September 26, 2013); and some sellers offer refunds entirely at their discretion, e.g., wine retailers such as WineBid.com. Shulman, et al. (2011) report that 19\% of all electronics are returned although there is no defect, and return rates as high as $35 \%$ for catalog retailers.

${ }^{6}$ Potential ways to make restarting with a clean record more difficult and costly have been discussed by some authors, such as Friedman and Resnick (2001), who term the issue as "cheap pseudonyms," and Dellarocas (2006). It has not been shown hitherto, however, how damaging cheap pseudonyms may be to the functioning of feedback systems.

${ }^{7}$ Mailath and Samuelson (2006) provide an extensive treatment of this literature.
} 
concerns the seller's ability to generate gains from trade (or cost of doing so), and learning occurs through observation of the seller's performance. We contribute to this literature by considering the possibility of communication and showing that it facilitates the learning process and enhances the reward from providing high quality. In this respect, our analysis extends the moral hazard model of reputation by Mailath and Samuelson (2001) where sellers exert high effort to separate from an inept type. In particular, we show that allowing communication in their setting strengthens the reputation effect, expedites learning, and thereby induces high effort even if there is no replacement of seller's type (see Section 6.3). Our work also contributes to the study of entry and exit of sellers (Tadelis, 1999; Mailath and Samuelson, 2001; Atkeson, Hellwig and Ordonez, 2012) by considering the possibility of re-entry under a new identity.

The existing literature on credibility is concerned with trustworthiness of communication. A main insight of the repeated cheap-talk literature (in particular, Sobel (1985) and Benabou and Laroque (1992)) is that the possibility that a message sender may be a "friend" with the same interests as the receiver can discipline other types of sender who have conflicting interests with the receiver. Our contribution lies in showing that effective communication may arise even when all types of sender (seller) have opposing interests with the receiver (buyer), if the type concerns the expected gains from trade with the sender. To our knowledge, Seidmann (1990) is the first to point out that effective communication may arise despite conflicting interests, if the receiver responds with a multi-dimensional action or has private information. Our approach is distinct in that we have a uni-dimensional response (the price) but in a repeated setting. Our work is also complementary to Ottaviani and Sorensen (2001, 2006a,b) and Gentskow and Shapiro (2006) who find that reputational concern may hinder communication if the uncertainty is on the accuracy of sender's information itself. ${ }^{8}$

Finally, our paper makes a methodological contribution to this literature in establishing the existence and uniqueness of equilibrium when Blackwell's condition for a contraction mapping does not apply, but value functions can be shown to be monotonic.

The rest of the paper is organised as follows. The next section describes the model and the core result. Section 3 illustrates some aspects of our reputation mechanism by means of a reduced, two-period model. Section 4 analyses the reputation mechanism and characterises the honest equilibrium, and its dynamic properties are discussed in Section 5. Section 6 presents the extensions to imperfect observation of quality and to voluntary refunds, as well as extensions where honest communication enhances welfare. Section 7 analyses the model with restart options. Section 8 concludes. The Appendix contains the key technical details and the Online Appendix contains additional proofs.

\footnotetext{
${ }^{8}$ Morris (2001) shows that when there is a possibility of conflict of interests, even a sender whose interests are perfectly aligned with the receiver's may distort reporting in order to enhance reputation.
} 


\section{Model and Core Result}

We study an infinite horizon model in which a long-lived seller sells one item to short-lived buyers in each period $t=1,2, \cdots$. The quality of the item for sale, denoted by $q_{t}$, is either good (i.e., $q_{t}=g$ ) or bad (i.e., $q_{t}=b$ ), and is an independent random draw from an identical distribution every period. We denote by $\theta=\operatorname{Pr}\left(q_{t}=g\right)$ the probability that the item's quality is good, which we refer to as the seller's ability or type. A seller is privately informed of his ability $\theta$, which is either high $(h)$ or low $(\ell)$ where $0<\ell<h<1$, and also privately observes the realised quality $q_{t}$ in each period.

Buyers are risk-neutral and do not observe the realised quality of the item before purchase. The item's quality is measured by the buyers' identical consumption value of the item, which we normalise as 1 and 0 for a good and bad quality item, respectively, i.e., $g=1$ and $b=0$. All buyers share the same initial common belief that the seller is of high ability (i.e., $\theta=h$ ) with probability $\mu_{1} \in(0,1)$ and is of low ability (i.e., $\theta=\ell$ ) with probability $1-\mu_{1}$. At the beginning of each period, all buyers will have the same information and therefore update their beliefs to the same posterior belief that the seller is of high ability with probability $\mu_{t} \in[0,1]$. We refer to this common belief $\mu_{t}$ as the seller's reputation.

In each period, after observing $q_{t}$, the seller publicly announces to all buyers a message $m_{t}$ from a discrete set $M$ of available messages, all of which are costless, non-binding and unverifiable, i.e., cheap talk. Then, the item is sold to one of the buyers at a price, $p_{t}$, that is equal to its expected quality based on the information shared by the buyers. ${ }^{9}$ The purchaser observes the item's true quality, $q_{t}$, and publicly reports it truthfully. We assume that $q_{t}$, albeit observable, is unverifiable ex-post, so no warranty contract is feasible on the realised quality.

Modelling a seller as above is in line with, for instance, online markets for collectibles and used goods, where the same seller repeatedly sells similar items of varying quality. Websites such as Amazon.com and PriceMinister.com specifically require the sellers to choose within a set of pre-codified levels (new, like new, good, fair, etc.) to describe the state of their items. The buyer's report is meant to capture feedback comments widely observed in online market platforms. Since the buyers are short-lived, they are indifferent between reporting truthfully and untruthfully. Nonetheless, we assume that they report truthfully because we are interested in the extent to which feedback comments may facilitate truthful communication.

A seller's strategy specifies, for each possible history, a probability distribution over the messages in $M$ according to which the seller will announce in the subsequent period, contingent on the seller's type and the realised quality of the item. Given the myopic nature of the buyers, payoff-relevant information contained in any history is fully captured by the seller's reputation level that it induces. Therefore, we focus on equilibria in Markov strategies that depend only on the prevailing reputation level $\mu_{t}$ and not the index $t$ of the period. Hence, we suppress the subscript $t$ unless necessary for clarity.

\footnotetext{
${ }^{9}$ This ensues if the buyers engage in Bertrand competition or the seller runs an efficient auction. It is also in line with Tadelis (1999), Mailath and Samuelson (2001) and Bar-Isaac (2003).
} 
Thus, a Markov strategy of a seller, denoted by $s^{*}$, is a collection of functions indexed by reputation level $\mu$, that specify a probability distribution over $M, s_{\mu}^{*}(\theta, q)$, for each pair of seller type $\theta \in\{h, \ell\}$ and item quality $q \in\{g, b\}$. A message is said to be used at reputation $\mu$ if it is announced with a positive probability for this reputation level, given $s^{*}$.

As said earlier, if a seller with reputation $\mu$ announces a message $m$, the item is traded at a price that is equal to the expected quality, which we denote by $p_{m}^{*}(\mu)$. It is determined by Bayes' rule from $s^{*}$ if $m$ is used at reputation $\mu$; otherwise, it is determined by the offequilibrium belief of the buyers. A price profile, denoted by $p^{*}$, is a collection of prices, one for every possible pair $(\mu, m)$, i.e., $p^{*}=\left\{p_{m}^{*}(\mu) \in \mathbb{R}_{+} \mid \mu \in[0,1], m \in M\right\}$.

Depending on the message announced, say $m$, and the item's quality, say $q$, the market updates the seller's reputation for the next period, which we denote by $\pi_{m q}^{*}(\mu) \in[0,1]$ : it is a posterior belief that the seller with reputation $\mu$ is of high ability in the contingency that he announced $m$ and the buyer reported the item's quality as $q \in\{g, b\}$. Again, given a Markov strategy $s^{*}, \pi_{m q}^{*}(\mu)$ is obtained by Bayes' rule provided that $m$ is used at this level of reputation. A transition rule, denoted by $\pi^{*}$, is defined as a collection of posterior beliefs, one for every possible tuple $(\mu, m, q)$, i.e., $\pi^{*}=\left\{\pi_{m q}^{*}(\mu) \in[0,1] \mid \mu \in[0,1], m \in M, q \in\{g, b\}\right\}$.

A price profile $p^{*}$ and a transition rule $\pi^{*}$ fully describe what the price will be in each period and how the seller's reputation will evolve in the next period, depending on the message announced by the seller and the item's quality in that period. Thus, starting from any reputation $\mu$, they allow us to calculate the payoff from any strategy $s$ a seller may employ, as the expected discounted sum of revenue stream:

$$
\mathbb{E}\left(\sum_{t=0}^{\infty} \delta^{t} p_{t} \mid \theta, p^{*}, \pi^{*}, s, \mu\right),
$$

where $\delta \in(0,1)$ is the discount factor.

We define a Markov equilibrium of the dynamic game described above, to consist of a Markov strategy $s^{*}$, a price profile $p^{*}$, and a transition rule $\pi^{*}$ such that

(i) $p^{*}$ and $\pi^{*}$ are obtained via Bayes' rule from $s^{*}$ whenever possible, and

(ii) for each $\theta \in\{h, \ell\}$ and $\mu \in[0,1]$, presuming that $p^{*}$ and $\pi^{*}$ prevail, the seller's payoff from $s^{*}$ is larger than or equal to that from any other strategy of the seller.

As is well known with cheap-talk games, the game described above has a "babbling" equilibrium in which messages play no role. This corresponds to the case that $s_{\mu}^{*}(\theta, q)$ is a uniform distribution over $M$ for all $\mu, \theta$, and $q$, so that announced messages carry no informational content. Consequently, regardless of the announced message, the equilibrium price is simply the expected quality of a seller's item given the prevailing reputation $\mu$, i.e.,

$$
\mathbb{E}(q \mid \mu)=\mu h+(1-\mu) \ell .
$$

Also, the seller's reputation is revised from $\mu$ based solely on the observed quality for the next period: depending on the observed quality $q$, it is

$$
\frac{\mu h}{\mu h+(1-\mu) \ell} \text { if } q=g, \quad \text { or } \quad \frac{\mu(1-h)}{\mu(1-h)+(1-\mu)(1-\ell)} \text { if } q=b \text {. }
$$


Thus, both the price of the item and the seller's reputation evolve as a martingale that results from pure learning on the seller's ability based only on the realised quality of the seller's item in each period. As the seller's message affects neither the price of the current period nor what will happen in the future, it is trivially optimal for the seller to "babble" in every period. Note that even without communication, observation of quality gives rise to a minimum amount of learning on the seller's type.

More interestingly, there are non-babbling equilibria in which messages affect the price and the seller's reputation, and as a result, effective communication arises in equilibrium. In this article, we are interested in the extent to which reputation motives induce trustworthy announcements on the quality of the product, at least by high ability sellers. Hence, in the following, we focus on equilibria with the property:

Condition $\mathbf{H}$ : At every $\mu>0$, an h-type seller uses disjoint sets of messages when quality is good and when it is bad.

This condition means that the buyers would be able to accurately infer the item's quality from the announced message if they knew the seller is of high ability. So, we call a Markov equilibrium that satisfies condition $\mathrm{H}$ an "honest equilibrium".

In the next two sections we study properties of honest equilibrium, and eventually establish the following core result of the paper.

Theorem 1 There exists an honest equilibrium if and only if $\delta \geq \delta_{h}:=1 /(h-\ell+1)$. All honest equilibria coincide on the equilibrium path.

Thus, an honest equilibrium always exists when the seller is patient enough, and is essentially unique. Moreover, the larger is the ability differential between the two types of seller, the more likely is trustworthy communication to arise.

To understand the nature of the result, it may be worth emphasising that both repeated interactions and adverse selection on the seller's ability need to be present in our environment for there to be any communication by either type of seller. As the market pays the expected quality of the item in any future period, in the absence of adverse selection (i.e., if the seller's type, $\theta$, is commonly known) the seller's expected revenue is the same (equal to $\theta$ ) in all future periods regardless of what happens in the current period. Consequently, the seller chooses a message myopically to maximize the current period's payoff, in which case all used messages fetch the same price, $\theta$, precluding any information transmission. Likewise, in the absence of repeated interactions, again the seller optimises the current period's payoff and as a result, no information may be transmitted by either type of seller, for the same reason (even if adverse selection is present). Note that this is because, unlike Sobel (1985) and Morris (2001), both types of seller have completely opposed interests to those of the buyers.

On the other hand, if both adverse selection and repeated interactions are present, the seller has an interest in convincing the buyers that his ability is high because this will fetch higher prices in the future on average. We show below that a seller may achieve this by honest cheap-talk communication which functions as an endogenous, dynamic signalling device that high ability sellers find less costly to adopt in the long-run. 


\section{A Reduced-Form Illustration}

Prior to a full-fledged analysis of the dynamic model described above, it is useful to examine a "reduced," two-period version of the game, that exhibits a number of key features that carry on to the infinite-horizon model. In the reduced model, the second period simply captures the feature that reputation is more valuable for a high ability seller, by a value function

$$
V_{\theta}^{*}\left(\mu_{2}\right)=\mu_{2} \cdot \theta \cdot v
$$

where $v>0$ is a constant that satisfies $\ell v<1$. So, the total payoff of a $\theta$-type seller is $p_{1}+\delta V_{\theta}^{*}\left(\mu_{2}\right)$ if the reputation level gets updated to $\mu_{2}$ after selling an item for a price $p_{1}$ in period 1 .

A key property of the value function (2) for our result is that

$$
V_{h}^{*}\left(\mu_{2}\right)-V_{h}^{*}(0)>V_{\ell}^{*}\left(\mu_{2}\right)-V_{\ell}^{*}(0) \quad \forall \mu_{2}>0 .
$$

This means that reputation premium is higher for an $h$-type seller than for an $\ell$-type seller, and provides the basis for more trustworthy behaviour by an $h$-type seller. Condition (3), which we refer to as the "sorting condition", is assumed in an ad hoc manner here but obtains endogenously in the main analysis of the next section.

We illustrate that an honest equilibrium exists in which an $h$-type seller always announces the quality truthfully in the first period of the reduced model. For expositional ease, we assume that only two messages are used in such equilibrium, $G$ and $B$, which are the messages that an $h$-type seller uses to truthfully announce a good and bad quality, respectively, in the first period.

First, note that the price is higher when $G$ is announced than when $B$ is announced in an honest equilibrium: this is so because otherwise, even an $\ell$-type seller would truthfully announce bad quality for both a higher price and a higher reputation update, in which case price should be lower when $B$ is announced. This further implies that an $\ell$-type seller announces good quality truthfully in an honest equilibrium as he can get both a higher price and a higher reputation next period.

If the quality is bad, however, he may lie by announcing $G$ for a short-term gain, say with probability $y$. Then, by Bayes' rule, the price in period 1 is $p_{B}^{*}(\mu)=0$ if $B$ is announced while it is

$$
p_{G}(\mu, y):=\frac{\mu h+(1-\mu) \ell}{\mu h+(1-\mu)(\ell+(1-\ell) y)} \geq \ell
$$

if $G$ is announced where $\mu \in(0,1)$ denotes the reputation level at the start of period 1 ; and the reputation level is updated to

$$
\pi_{B b}(\mu, y):=\frac{\mu(1-h)}{\mu(1-h)+(1-\mu)(1-\ell)(1-y)}
$$

after a truthful announcement of $B$ and collapses to $\pi_{G b}^{*}(\mu)=0$ after lying.

Upon drawing a bad quality item, therefore, it is optimal for a seller to lie if the shortterm gain in current payoff, $p_{G}(\mu, y)-p_{B}^{*}(\mu)=p_{G}(\mu, y)$, exceeds the long-term loss from 
spoiled reputation, $\delta\left[V_{\theta}^{*}\left(\pi_{B b}(\mu, y)\right)-V_{\theta}^{*}(0)\right]$; and to announce truthfully if the opposite is true. Hence, the equilibrium value of $y$, denoted by $y^{*}(\mu)$, must satisfy:

$$
y^{*}(\mu)\left\{\begin{array}{ccc}
=1 & \Longrightarrow & p_{G}\left(\mu, y^{*}(\mu)\right) \geq \delta\left[V_{\ell}^{*}\left(\pi_{B b}\left(\mu, y^{*}(\mu)\right)\right)-V_{\ell}^{*}(0)\right] \\
\in(0,1) & \Longrightarrow & p_{G}\left(\mu, y^{*}(\mu)\right)=\delta\left[V_{\ell}^{*}\left(\pi_{B b}\left(\mu, y^{*}(\mu)\right)\right)-V_{\ell}^{*}(0)\right] \\
=0 & \Longrightarrow & p_{G}\left(\mu, y^{*}(\mu)\right) \leq \delta\left[V_{\ell}^{*}\left(\pi_{B b}\left(\mu, y^{*}(\mu)\right)\right)-V_{\ell}^{*}(0)\right]
\end{array}\right.
$$

To pin down the value of $y^{*}(\mu)$, observe that as an $\ell$-type seller lies more, the current price decreases while truth-telling becomes a stronger signal of $h$-type. Thus,

(i) the short-term gain, $p_{G}(\mu, y)$, decreases in $y$, but

(ii) the long-term loss of an $\ell$-type seller, $\delta\left[V_{\ell}^{*}\left(\pi_{B b}(\mu, y)\right)-V_{\ell}^{*}(0)\right]$, increases in $y$.

So, the net gain from lying falls in $y$ and obtains a minimum level of $p_{G}(\mu, 1)-\delta\left[V_{\ell}^{*}(1)-\right.$ $\left.V_{\ell}^{*}(0)\right]$ when $y=1$. If this level is positive, an $\ell$-type seller must lie for sure, i.e., $y^{*}(\mu)=1$. As $p_{G}(\mu, 1)$ increases in $\mu$, denoting

$$
\Delta:=V_{\ell}^{*}(1)-V_{\ell}^{*}(0)
$$

this is the case for all $\mu \geq \bar{\mu}$ where the threshold is given by

$$
\bar{\mu}:=\min \left\{\mu \in[0,1] \mid p_{G}(\mu, 1) \geq \delta \Delta\right\}<1 .
$$

As $p_{G}(1,1)=1$ and $\Delta=\ell v<1$ (by assumption in this section), we have $\bar{\mu}<1$. Moreover, $\bar{\mu}=0$ if $p_{G}(0,1)=\ell \geq \delta \Delta$.

Consider the case that $\ell<\delta \Delta$ so that $\bar{\mu}>0$. For every positive $\mu<\bar{\mu}$, the net gain from lying is negative when $y=1$, but positive when $y=0$ as $p_{G}(\mu, 0)=1>\delta \Delta$. Hence, it assumes 0 at a unique value of $y$ that solves

$$
p_{G}(\mu, y)=\delta\left[V_{\ell}^{*}\left(\pi_{B b}(\mu, y)\right)-V_{\ell}^{*}(0)\right]
$$

as illustrated in Figure 1 (a) below. This solution is the unique equilibrium value $y^{*}(\mu) \in$ $(0,1)$, at which lying and telling the truth are equivalent for an $\ell$-type seller.

Figure 1

(a)

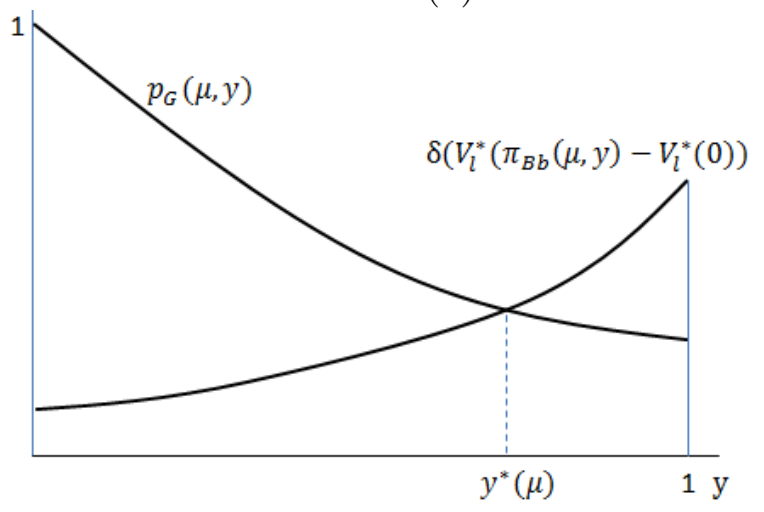

(b)

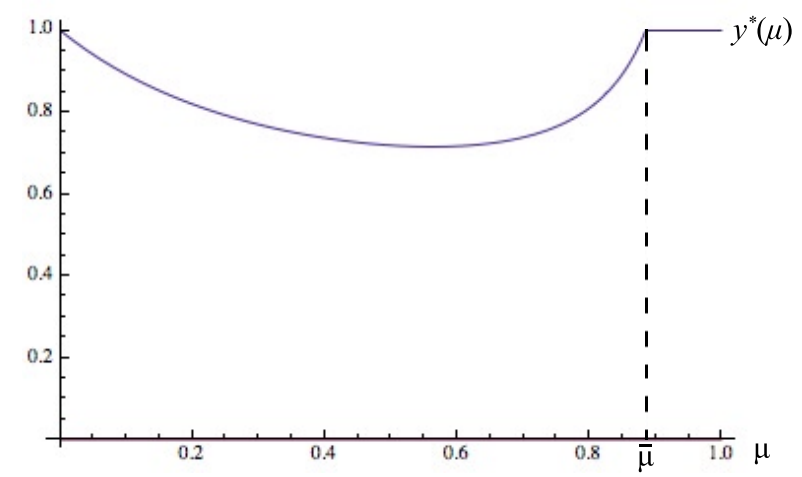


For every $y<1$, we have $p_{G}(\mu, y) \geq \ell$ and $\lim _{\mu \rightarrow 0} \pi_{B b}(\mu, y)=0$. Hence, the solution $y^{*}(\mu)$ to (9) converges to 1 as $\mu \rightarrow 0$, so that $p_{G}\left(\mu, y^{*}(\mu)\right)$ converges to $\ell$. Thus, it is further implied by $(9)$ that $\pi_{B b}\left(\mu, y^{*}(\mu)\right)$ converges to some positive value $\pi_{B b}^{*}\left(0^{+}\right)>0$ as $\mu \rightarrow 0$, such that $\delta\left[V_{\ell}^{*}\left(\pi_{B b}^{*}\left(0^{+}\right)\right)-V_{\ell}^{*}(0)\right]=\ell$. Intuitively, to balance the short-term gain, a substantial reputation gain should be warranted from truth-telling, which is feasible only if an $\ell$-type seller lies almost surely at very low levels of reputation. As $y^{*}$ is continuous and assumes unity for $\mu \geq \bar{\mu}$ as explained above, $y^{*}(\mu)$ is non-monotonic as illustrated in Figure 1 (b) for the case that $h=0.5, \ell=0.3, \delta=0.95$ and $v=3$.

The unique optimal strategy $y^{*}(\mu)$ identified above is valid so long as $V_{\ell}^{*}$ is continuous and strictly increasing and $\delta \Delta<1$, as stated below for use in the next section.

Lemma 1 Suppose that $V_{\ell}^{*}:[0,1] \rightarrow \mathbb{R}$ is continuous and strictly increasing and $\delta \Delta<1$. There is a unique function $y^{*}:(0,1) \rightarrow[0,1]$ that satisfies $(6)$, and the following holds.

(a) $y^{*}(\mu)$ is strictly positive and continuous at all $\mu \in(0,1)$.

(b) $y^{*}(\mu)=1$ for $\mu \geq \bar{\mu}$, and if $\delta<\ell / \Delta$ then $y^{*}(\mu)<1$ for $\mu \in(0, \bar{\mu})$ with $\lim _{\mu \rightarrow 0} y^{*}(\mu)=1$.

To establish that an honest equilibrium exists, it remains to verify that an $h$-type seller prefers to announce quality truthfully in period 1 at all levels of $\mu \in(0,1)$. Observe that the sorting condition, (3), implies that an $h$-type seller has more incentive to be truthful than an $\ell$-type seller for all $\mu$. Because the short-term gain from lying when quality is bad, $p_{G}^{*}(\mu)$, is the same for both types, an $h$-type seller must find it optimal to truthfully announce a bad quality whenever an $\ell$-type seller is indifferent between lying and not, which is the case when $\mu \in(0, \bar{\mu})$. By the same token, whenever an $\ell$-type seller prefers to tell the truth, which is the case when quality is good, so does an $h$-type seller.

In the remaining case that $\mu \geq \bar{\mu}$ and quality is bad, telling the truth reveals high ability and an $h$-type seller would find it optimal to do so if the long-term loss from lying, $\delta\left(V_{h}^{*}(1)-V_{h}^{*}(0)\right)$, exceeds the short-term gain, $p_{G}^{*}(\mu)$. As $p_{G}^{*}(\mu)$ reaches a maximum value of 1 at $\mu=1$, this condition is satisfied for all $\mu \geq \bar{\mu}$ if and only if $\delta\left(V_{h}^{*}(1)-V_{h}^{*}(0)\right) \geq$ $p_{G}^{*}(1)=1$. In our two-period model where $V_{h}^{*}(1)-V_{h}^{*}(0)=h v$, therefore, we conclude that an honest equilibrium exists for any initial positive reputation provided that $\delta h v>1$.

We have illustrated in a reduced two-period model that, despite the conflicts of interest between seller and buyer, effective communication may arise when the seller's ability is private information, provided that the future benefit from enhanced reputation is larger for a high ability seller. In a nutshell, a high ability seller behaves honestly to signal ability and raise reputation for future benefit, which works because his low ability counterpart, who would derive smaller benefit from enhanced reputation, does not behave as honestly.

A key remaining question is how one may justify the disparity in the value of reputation between the two types of seller. In the next section we show that in a fully dynamic model, the very expectation that an honest equilibrium will prevail in the future is sufficient to induce the sorting condition (3) endogenously, thus furnishing the right incentives for the seller to behave as expected in the equilibrium. 


\section{Honest Equilibrium}

We now turn to a full-fledged dynamic analysis and show that our core insights naturally prevail in the infinite-horizon model described in Section 2. Moreover, mutual determination of equilibrium strategy and value functions pins down what happens on the equilibrium path uniquely, generating a number of clear predictions on dynamics and testable comparative statics results.

As a seller's equilibrium strategy determines the value function and vice versa, equilibrium value functions are fixed points of a mapping suitably defined on a space of possible value functions. To characterise such an equilibrium, we first establish a set of properties equilibrium strategy and value functions should satisfy (Section 4.1); then based on these findings, we devise an appropriate mapping from a space of possible value functions to itself, in order to finally establish existence and uniqueness of a fixed point (Section 4.2). As an $h$-type seller is always truthful by definition in an honest equilibrium, we focus on the equilibrium strategy and the value function of an $\ell$-type seller first, and then discuss the incentives of an $h$-type seller to disclose quality truthfully (Section 4.3). We present the analysis heuristically here and provide full details in the Appendix.

\subsection{Properties of $\ell$-type seller strategy and value function}

We continue to present our analysis postulating a binary message space $M=\{G, B\}$, where $G$ and $B$ are the messages that an $h$-type seller with reputation $\mu>0$ announces when the realised quality of the item is good and bad, respectively, in an honest equilibrium. Thus, we say that a seller of either type tells the truth if the seller announces $G$ when $q=g$ or announces $B$ when $q=b$, and that the seller lies otherwise. Then, Condition H means that an $h$-type seller always tells the truth when $\mu>0$.

The restriction to a binary message space is innocuous because in any honest equilibrium, as we show in Appendix A.1, all messages that an $h$-type seller sends for a given $q \in\{g, b\}$ lead to the same price and posterior beliefs, and an $\ell$-type seller would never reveal the type by sending a message not sent by an $h$-type seller. An implication of this result is that in any honest equilibrium the seller is unable to signal ability separately from the item's quality.

Consider an honest equilibrium (supposing one exists) of the infinite-horizon model of Section 2, represented by a Markov announcement strategy $s^{*}$, a price profile $p^{*}$ and a transition rule $\pi^{*}$. This determines a value function, denoted by $V_{\theta}^{*}:[0,1] \rightarrow \mathbb{R}$, for each type $\theta \in\{h, \ell\}$ such that $V_{\theta}^{*}(\mu)$ is the expected discounted sum of the future income stream starting from a period with reputation level $\mu \in[0,1]$ in this equilibrium.

Once the reputation level falls to $\mu=0$, an $\ell$-type seller cannot lift it above 0 because Bayes-updated reputation level is invariably 0 after any message that is announced with a positive probability in equilibrium. Therefore, an $\ell$-type seller with reputation $\mu=0$ announces the message that gives the highest price regardless of $q$, which implies that the seller gets the same price regardless of $q$ in every period, equal to the expected quality of 
the item given $\mu=0$, i.e., $\ell$. Consequently,

$$
V_{\ell}^{*}(0)=\sum_{t=0}^{\infty} \delta^{t} \ell=\frac{\ell}{1-\delta} .
$$

For expositional ease, we adopt an innocuous convention that an $\ell$-type seller announces $G$ regardless of $q$ when $\mu=0$.

Observe that an $\ell$-type seller with positive reputation $\mu>0$, upon drawing a good quality item, should truthfully announce $G$ by the same reasoning as that leading to the same conclusion in the two-period model of the previous section. Therefore, we characterise equilibrium strategy of an honest equilibrium by determining the probability with which an $\ell$-type seller with reputation $\mu$ lies when the quality is bad, denoted by $y^{*}(\mu) \in[0,1]$, and an $h$-type seller's announcement strategy in the off-equilibrium contingency that $\mu=0$.

Thus, a price profile, $p^{*}$, and a transition rule, $\pi^{*}$, are determined in the same manner as explained in the previous section, which we recap here. Since even an $\ell$-type seller never lies when $q=g$, the prices are

$$
p_{B}^{*}(\mu)=0 \quad \text { and } \quad p_{G}^{*}(\mu)=p_{G}\left(\mu, y^{*}(\mu)\right) \text { for all } \quad \mu \in[0,1]
$$

where $p_{G}(\mu, y)$ is defined as in (4). ${ }^{10}$ The seller's reputation level gets updated as

$$
\pi_{G g}^{*}(\mu)=\frac{\mu h}{\mu h+(1-\mu) \ell}, \pi_{B b}^{*}(\mu)=\pi_{B b}\left(\mu, y^{*}(\mu)\right), \pi_{G b}^{*}(\mu)=0 \quad \forall \mu \in[0,1]
$$

whenever well-defined by Bayes' rule, where $\pi_{B b}(\mu, y)$ is defined in (5). In all contingencies where Bayes' rule does not apply, we postulate that the reputation is updated to the lowest level, that is, $\pi_{B g}^{*}(\mu)=0$ for all $\mu$ and $\pi_{G b}^{*}(1)=\pi_{B b}^{*}(0)=0 .{ }^{11}$ These specify payoffs for irrelevant or zero probability events and are innocuous for our purpose because, as we show in Appendix A.2, any honest equilibrium continues to be one when these posteriors are imposed on the off-equilibrium contingencies.

We established the value at $\mu=0$ in equation (10) above. Now, consider an $\ell$-type seller with the maximum reputation $\mu=1$. If it was optimal for this seller to announce even bad quality honestly, his expected equilibrium payoff would be $\ell /(1-\delta)$ because then by telling the truth in every period, he would get a price $p_{G}^{*}(1)=1$ with probability $\ell$ and $p_{G}^{*}(0)=0$ with probability $1-\ell$. But this is also this seller's continuation value $V_{\ell}^{*}(0)$ after lying and revealing a low type. This implies that an $\ell$-type seller must lie for sure upon drawing a bad quality, i.e., $y^{*}(1)=1$. As $\pi_{G b}^{*}(1)=0$, we have $V_{\ell}^{*}(1)=1+\delta\left(\ell V_{\ell}^{*}(1)+(1-\ell) V_{\ell}^{*}(0)\right)$ so that

$$
V_{\ell}^{*}(1)=V_{\ell}^{*}(0)+\frac{1-\ell}{1-\delta \ell} \Longleftrightarrow \Delta=V_{\ell}^{*}(1)-V_{\ell}^{*}(0)=\frac{1-\ell}{1-\delta \ell}<1 .
$$

\footnotetext{
${ }^{10}$ Note that $p_{B}^{*}(0)$ is not defined by Bayes' rule. We set it at 0 for expositional ease although any value less than $\ell$ would do.

${ }^{11}$ The interpretation of $\pi_{G b}^{*}(1)=0$ is that buyers, having classified the seller as $h$-type with probability 1 based on some past records, would reconsider their interpretation of the records upon (hypothetical) arrival of new evidence inconsistent with this classification. That $\pi_{B b}^{*}(0)=0$ implies that once the reputation collapses to nil due to a lie, even an $h$-type seller would be trapped there forever. We will relax the stipulation $\pi_{B b}^{*}(0)=0$ for the extended models analysed in the Online Appendix to allow for a different value of $V_{h}^{*}(0)$.
} 
That the value of a maximum reputational gain for an $\ell$-type seller, $\Delta$, is smaller than the maximum price differential, $g-b=1$, can be understood as follows: an $\ell$-type seller has only one chance to lie and cash in reputation, and as the chance may not come immediately, $\Delta$ falls short of the maximum price differential which is 1 .

In an honest equilibrium, the value function $V_{\ell}^{*}$ is the optimised discounted sum of expected income stream of an $\ell$-type seller when the buyers expect the probability of lying to be $y^{*}$ and thus, the prices and posterior beliefs are given by (11) and (12). In addition to the extreme values obtained above, it exhibits the following property:

Lemma 2 In any honest equilibrium, $V_{\ell}^{*}(\mu)$ is continuous and strictly increasing in $\mu \in$ $[0,1]$.

As a formal proof requires developing some analytic building blocks, we defer it to the Appendix A.3. While the proof is involved, the result essentially reflects the straightforward intuition that as an $h$-type seller always tells the truth, announcement of good quality gains credibility and raises the seller's reputation. As a result, a seller of either type is facing a better income prospect at a higher reputation level.

Recall from the previous section that an $\ell$-type seller with a bad quality item will lie if the short-term gain, $p_{G}^{*}(\mu)$, exceeds the long-term loss, $\delta\left[V_{\ell}^{*}\left(\pi_{B b}^{*}(\mu)\right)-V_{\ell}^{*}(0)\right]$, and consequently, the equilibrium lying probability $y^{*}(\mu)$ should satisfy the condition (6). As $\delta \Delta<1$ by (13) and $V_{\ell}^{*}$ is continuous and strictly increasing by Lemma 2, Lemma 1 characterises the properties of $y^{*}(\mu)$ in an honest equilibrium of the infinite-horizon model as well. Hence, $y^{*}(\mu)=1$ if $\mu$ is above the threshold $\bar{\mu}$ defined in (8) because for such values of $\mu$ the short-term gain exceeds the maximum possible long-term loss, $\delta \Delta$, regardless of $y$. For $\mu \in(0, \bar{\mu}), y^{*}(\mu)$ is strictly between 0 and 1 and changes continuously, converging to 1 as $\mu$ tends to 0 and to $\bar{\mu}$.

Moreover, since the short-term gain $p_{G}(\mu, y)$ approaches 1 as $y$ tends to 0 while the long-term loss is bounded above by $\delta \Delta<1$, the condition that these two values must be equal when $y^{*}(\mu) \in(0,1)$ imposes a uniform lower bound on $y^{*}(\mu)$ as proved in the Appendix (proof of Lemma 5):

$$
y^{*}(\mu)>\hat{y}:=\frac{h-\ell}{1-\ell} \text { for all } \mu .
$$

In other words, there is a uniform upper bound on the extent of honesty by an $\ell$-type seller. As will be discussed below, this has important consequences on equilibrium dynamics.

Summarising the properties of $y^{*}$ obtained above, we have established that

$y^{*}$ is continuous, $y^{*}(\mu)>\hat{y}$ for all $\mu$, and $y^{*}(\mu)=1$ if and only if $\mu \in\{0\} \cup[\bar{\mu}, 1]$.

Lastly, recall from (8) that $\bar{\mu}>0$ if and only if $\delta \Delta>\ell=p_{G}(0,1)$. This implies that $\ell$-type sellers may announce a bad quality truthfully only if they are patient enough, i.e., if

$$
\delta>\delta_{\ell}:=\frac{\ell}{1-\ell+\ell^{2}} .
$$

In the opposite case (i.e., if $\delta \leq \delta_{\ell}$ ), an $\ell$-type seller always announces $G$ regardless of quality in any honest equilibrium. 


\subsection{Existence and uniqueness of $\ell$-type seller strategy and value function}

We have shown $y^{*}(\mu)>0$ above, so that announcing $G$ is an optimal strategy for an $\ell$-type seller when $q=b$, as well as when $q=g$. Hence, an $\ell$-type seller's equilibrium value function $V_{\ell}^{*}$ satisfies the recursive formula when he always announces $G$ :

$$
V_{\ell}^{*}(\mu)=p_{G}^{*}(\mu)+\delta\left(\ell V_{\ell}^{*}\left(\pi_{G g}^{*}(\mu)\right)+(1-\ell) V_{\ell}^{*}(0)\right) .
$$

Equilibrium requires (17) and optimality of $y^{*}(\mu)$ in every period given the price profile $p^{*}$, the transition rule $\pi^{*}$, and the next period's value given by $V_{\ell}^{*}(\cdot)$. To identify an honest equilibrium, therefore, we look for a pair of functions $y^{*}$ and $V_{\ell}^{*}$ that satisfy these conditions.

We start with the set, denoted by $\mathcal{F}$, of all functions that satisfy the properties obtained above for $V_{\ell}^{*}: \mathcal{F}$ is the set of all continuous and strictly increasing functions $V$ on $[0,1]$ with $V(0)=\ell /(1-\delta)$ and $V(1)=V(0)+\Delta$. Given an arbitrary "candidate" value function $V \in \mathcal{F}$, we define an $\ell$-type seller's "optimal response" relative to $V$ for each $\mu \in[0,1]$, denoted by $y_{V}(\mu)$, as the lying probability of an $\ell$-type seller with a bad quality item that is optimal when the price and reputation updating reflect it correctly:

$$
y_{V}(\mu) \in \arg \max _{0 \leq y \leq 1} y\left(p_{G}\left(\mu, y_{V}(\mu)\right)+\delta V(0)\right)+(1-y) \delta V\left(\pi_{B b}\left(\mu, y_{V}(\mu)\right)\right) .
$$

Note that determining $y_{V}(\mu)$ in this manner is equivalent to the condition (6) that determines the first period equilibrium lying probability relative to the value function given as (2) in the reduced, two-period model of Section 3. As discussed there, since $V$ is continuous and increasing in $\mu$ and $\delta \Delta<1$, the value of $y_{V}(\mu)$ is uniquely determined as

$$
y_{V}(\mu)=\left\{\begin{array}{lll}
1 & \text { if } & p_{G}(\mu, 1)>\delta\left[V\left(\pi_{B b}(\mu, 1)\right)-V(0)\right]=\delta \Delta \\
y & \text { s.t. } & p_{G}(\mu, y)=\delta\left[V\left(\pi_{B b}(\mu, y)\right)-V(0)\right], \text { otherwise. }
\end{array}\right.
$$

As $y_{V}(\mu)>0$ for all $\mu$, in conformity with the recursive formulation, we define an operator, $T$, that revises the candidate value function using the optimal response as

$$
T(V)(\mu):=p_{G}\left(\mu, y_{V}(\mu)\right)+\delta\left(\ell V\left(\pi_{G g}^{*}(\mu)\right)+(1-\ell) V(0)\right) .
$$

Note that $T(V)(0)=V(0)$ and also that $T(V)(1)=V(1) \cdot{ }^{12}$ In addition, we prove in the Appendix A.4 that given that $p_{G}, \pi_{B b}$ and $V$ are continuous and strictly increasing in $\mu$, so is $T(V)$. Therefore, $T$ is a well-defined operator from $\mathcal{F}$ to itself.

An honest equilibrium value function $V_{\ell}^{*}$ is a fixed point of $T$, and the equilibrium strategy $y^{*}$ is an optimal response relative to $V_{\ell}^{*}$ as defined in (18). Thus, such $y^{*}$ and $V_{\ell}^{*}$ exist if the operator $T$ has a fixed point. Indeed, we show that

Proposition 1 There exists a unique fixed point $V_{\ell}^{*}$ of $T$ in $\mathcal{F}$.

\footnotetext{
${ }^{12}$ This is because $\pi_{B b}(0, y)=0$ for all $y<1$ implies $y_{V}(0)=1$, and $p_{G}(0,1)=\ell$ while $p_{G}\left(1, y_{V}(1)\right)=1$.
} 
In our model $T$ is not non-decreasing in $V$ because $y_{V}$ is non-monotonic in $V$, so our result is unobtainable by applying Blackwell's Theorem as is often done in related studies. $^{13}$ Instead, we show existence by a suitable application of Fan-Glicksberg Fixed Point Theorem, and uniqueness by exploiting the properties of a fixed point of $T$, as elaborated in a proof provided in the Appendix A.4.

\subsection{Optimality of $h$-type seller strategy}

We have established that there uniquely exist an announcement strategy $y^{*}$ and a value function $V_{\ell}^{*}$ that satisfy the equilibrium conditions for an $\ell$-type seller. Therefore, an honest equilibrium exists if an $h$-type seller finds it optimal to tell the truth at all $\mu>0$, presuming that the market reacts according to the price profile $p^{*}$ and the transition rule $\pi^{*}$ induced by the strategy $y^{*}$. We verify that this is indeed the case if $\delta \geq \delta_{h}$.

Let $V_{h}^{*}(\mu)$ denote the maximum expected discounted sum of the income stream that an $h$-type seller with a reputation $\mu$ can obtain, presuming that the market reacts according to $p^{*}$ and $\pi^{*}$. At the minimal reputation $\mu=0$, an $h$-type seller would announce $G$ regardless of quality because $p_{G}^{*}(0)=\ell>p_{B}^{*}(0)=0$ and he cannot escape from the minimal reputation according to $(12)$. Thus, $V_{h}^{*}(0)=\ell /(1-\delta)$.

As in the reduced-form illustration of Section 3, a key factor for the optimality of an $h$-type seller's strategy is the sorting condition which holds in the current case as well:

Proposition $2 V_{h}^{*}(\mu)-V_{h}^{*}(0)>V_{\ell}^{*}(\mu)-V_{\ell}^{*}(0)$ for all $\mu>0$.

This result, which we prove in the Appendix A.5, stems from the fact that by definition $V_{h}^{*}(\mu)$ is no lower than the expected discounted sum of an $h$-type seller's income stream from playing the equilibrium strategy of an $\ell$-type seller. The value from this strategy is higher for an $h$-type seller because an $h$-type seller will have more good draws on average than an $\ell$-type seller before drawing a bad quality item after which the reputation collapses to nil. As $V_{h}^{*}(0)=V_{\ell}^{*}(0)$, Proposition 2 ensues.

As the value of maintaining reputation is higher for an $h$-type seller than for an $\ell$ type seller due to the sorting condition, we can replicate the argument in Section 3 for the optimality of $h$-type seller's strategy. An $h$-type seller must find truth-telling optimal whenever an $\ell$-type seller finds it no worse than lying, which is the case if $q=g$ or if $g=b$ and $\mu \leq \bar{\mu}$. When $g=b$ and $\mu>\bar{\mu}$, as $p^{*}(\mu) \leq 1$ and $\pi_{B b}^{*}(\mu)=1$, truth-telling is optimal for an $h$-type seller if $\delta\left(V_{h}^{*}(1)-V_{h}^{*}(0)\right) \geq 1$. Given that $V_{h}^{*}(1)=h /(1-\delta)$, this last condition holds when $\delta \geq \delta_{h}$, leading to the following conclusion:

Proposition 3 There is an honest equilibrium if and only if $\delta \geq \delta_{h}$.

The proposition is proved in the Appendix A.6. As the equilibrium strategy of an $\ell$-type seller, $y^{*}$, is unique due to Proposition 1 and (18), all honest equilibria coincide on the equilibrium path. This establishes Theorem 1.

\footnotetext{
${ }^{13}$ E.g., Benabou and Laroque (1992), Morris (2001) and Bar-Isaac (2003) use Blackwell's Theorem.
} 


\section{$5 \quad$ Reputation and price dynamics}

Recall that in the babbling equilibrium, both the price and reputation move as a martingale depending only on the delivered quality. A seller's reputation approaches that seller's true ability asymptotically, and the price does not reflect information about the current quality beyond what is implied by the prevailing reputation. In contrast, in the honest equilibrium seller's announcements carry further information and affect price and reputation dynamics, as we elaborate below.

Price dynamics. In the honest equilibrium, the prices reflect the realised quality in each period, albeit less than perfectly until the reputation reaches 1 . Because

$$
h p_{G}^{*}(\mu)<\mathbb{E}(q \mid \mu)
$$

as is easily verified from (1), (4) and (14), communication on quality comes at a short-run cost for high ability sellers. This stems from the fact that high ability sellers sacrifice in the short-run to convince the market of their high ability for future benefits.

Enhanced reputation may have conflicting effects on the price $p_{G}^{*}$ because greater confidence that the seller is of $h$-type may be undermined by an increase in $y^{*}(\mu)$. We show however in the Appendix (Lemma 5) that

$$
p_{G}^{*}(\mu) \text { increases in } \mu \text {. }
$$

So, increased reputation fosters trust in communication in our model, i.e., buyers put more weight on the word of a seller who is perceived as more capable of delivering good quality.

Reputation dynamics. As is elaborated in the Appendix (Lemma 5), the lower bound on $y^{*}$ specified in (14) is obtained from the following property of honest equilibrium:

$$
\pi_{B b}^{*}(\mu)>\mu \quad \forall \mu>0 .
$$

In conjunction with the fact that $\pi_{G g}^{*}(\mu)>\mu$, this means that honest behaviour always enhances a seller's reputation. That is, unlike in the babbling equilibrium where bad quality always dents reputation, truthfully announced bad quality is interpreted as "good news" in the honest equilibrium and enhances reputation.

Thus, a seller's reputation increases until one of two events occurs: $i$ ) bad quality is falsely claimed as good, in which case the seller's low ability is revealed; or $i i$ ) bad quality is truthfully announced when the reputation is above $\bar{\mu}$, in which case the seller's ability is revealed to be high. As such, a seller's type is revealed within a finite time with probability 1 , because the reputation level goes above $\bar{\mu}$ within a finite number of periods (unless it collapses to zero due to a false announcement), after which the seller's type gets revealed at the first draw of bad quality.

The feature that a seller's reputation increases until it vanishes with a single lie, albeit simplified, is consistent with the observation that active sellers in online marketplaces tend to be concentrated on the high end of the grade distribution. ${ }^{14}$ According to our analysis,

\footnotetext{
${ }^{14}$ See Cabral and Hortacsu (2010).
} 
a few bad grades suffice to undermine the viability of a seller (Cf. Section 6 on sellers who are imperfectly informed and Section 7 on endogenous participation of sellers).

More honesty slows down learning. As pointed out earlier, a seller's message in each period cannot carry information on the item's quality unless it also carries information on the seller's ability. The former information is reflected in price and the latter in reputation. Hence, an intrinsic link exists between price and reputation dynamics in the honest equilibrium.

In particular, our analysis uncovers a trade-off that a seller's announcement strategy induces between short-term transmission of information on quality and the speed of learning on the seller's type: When an $\ell$-type seller behaves more honestly, the price reflects the quality better as a direct consequence, but the inference on the seller's type from that seller's announcement is weakened because the two types behave more similarly.

This trade-off has interesting implications on the effect of discount rate changes. As intuition suggests and we show in the Appendix B, more patient sellers are more trustworthy at all levels of reputation:

Proposition 4 Represent $\bar{\mu}$ and $y^{*}(\mu)$ as $\bar{\mu}(\delta)$ and $y^{*}(\mu \mid \delta)$ to reflect their dependence on $\delta$. If $\delta_{\ell}<\delta<\delta^{\prime}<1$, then $\bar{\mu}(\delta)<\bar{\mu}\left(\delta^{\prime}\right)$ and $y^{*}\left(\mu \mid \delta^{\prime}\right)<y^{*}(\mu \mid \delta)$ for every $0<\mu<\bar{\mu}\left(\delta^{\prime}\right)$.

A consequence of Proposition 4 is that prices reflect the true quality more accurately in every period, but the true ability of the seller is revealed at a slower pace.

Who benefits from honesty? For expositional clarity we analysed a baseline model in which trade is always efficient, so the total welfare is the same across equilibria. Below we discuss who benefits from communication in this baseline model. In the next section, on the other hand, we present possible extensions in which the total welfare increases and consumers benefit from communication.

As the unconditional expected price reflects the expected quality of the item, a seller's ex-ante payoff in an honest equilibrium, $\mu_{1} V_{h}^{*}\left(\mu_{1}\right)+\left(1-\mu_{1}\right) V_{\ell}^{*}\left(\mu_{1}\right)$, is equal to $\mathbb{E}\left(q \mid \mu_{1}\right) /(1-$ $\delta)$ which is also a seller's ex-ante payoff in the babbling equilibrium. Which type of seller is better-off in an honest equilibrium than in the babbling equilibrium? The answer is not trivial. To see this point notice that an $h$-type seller induces posteriors $\pi_{G g}^{*}(\mu)$ and $\pi_{B b}^{*}(\mu)>\mu$ in an honest equilibrium depending on the realised quality, while he induces posteriors $\pi_{G g}^{*}(\mu)$ and $\pi_{B b}(\mu, 0)<\mu$ in the babbling equilibrium. Hence, high ability sellers benefit from uniformly better posteriors in an honest equilibrium. But, (20) means that their expected short-run price is lower in an honest equilibrium at every reputation level. Thus, the comparison depends on whether lower short-run prices are compensated by faster appreciation of reputation.

However, we verify that $h$-type sellers are better off in an honest equilibrium at least for the case that the reputation level is not too low, provided that they are patient enough.

Proposition 5 An h-type seller is better-off in an honest equilibrium than in the babbling equilibrium if $\mu_{1}$ exceeds a threshold $\mu^{*}(h, \ell)<1$ and $\delta$ is large enough, where $\mu^{*}(h, \ell) \rightarrow 0$ as $\ell$ tends either to 0 or to $h$, or $h$ tends to 1 . 
In the proof provided in Appendix B, we bound $\mu^{*}(h, \ell)$ from above by quite low levels typically, for example, below 0.2 for $\ell, h<0.7$. We conjecture that $\mu^{*}(h, \ell)$ is nil or very small for all $(h, \ell)$ based on various simulation results (available upon request).

\section{Extensions and discussions}

\subsection{Imperfect observation of product quality}

We assumed perfect observation of quality until now, so that the seller's honesty is detected without error ex post. This simplifies analysis and exposition but at a cost of reduced realism. We show that our main results extend to more realistic cases, in particular, when the seller's observation of quality is subject to a small noise.

Such a noise in observation creates an issue in sustaining an $h$-type seller's incentive to be truthful at the upper end of reputation, because a false claim of bad quality as good would be attributed to an observational error rather than to a deliberate lie when the seller is believed almost surely to be of an $h$-type. The crumbling of incentives at the upper end of reputation has knock-on effects on lower ranges, undermining trustworthy behaviour across the board. This issue has been studied in the literature (Cripps, et al., 2004) and several authors showed that it may be resolved if the uncertainty about the type is continually renewed by stochastic and unobservable replacement of the player (Mailath and Samuelson (2001) and Phelan (2006)), because then extremely high reputation levels do not arise. We also adopt this approach.

Given that the source of the problem stems from the desire to exploit the possibility of erroneous observation of bad quality as good, rather than the other way around, we postulate that there is no observation error of good quality for expositional ease (our result does not rely on this simplification). Specifically, we augment the model in Section 2 in the following two respects:

a) In every period, the seller receives a signal $\hat{q}_{t} \in\{g, b\}$ such that $\operatorname{Pr}\left(\hat{q}_{t}=g \mid q_{t}=g\right)=$ $1>\operatorname{Pr}\left(\hat{q}_{t}=b \mid q_{t}=b\right)=\alpha$.

b) In every period, the seller is replaced by another seller of the other type with a small, exogenous probability $1-\beta$, where $\beta<1$ is close to 1 .

Hence, announcing $B$ after observing $\hat{q}_{t}=g$ is detected as a lie for sure, but announcing $G$ after observing $\hat{q}_{t}=b$ could be attributed to an erroneous signal. Compared to the baseline model, the key difference in the augmented model is that, if an announcement of $G$ by a seller with reputation $\mu$ turns out to be false, Bayes' rule prescribes a posterior that is equal not to zero but to

$$
\hat{\pi}_{G b}(\mu, y):=(2 \beta-1) \frac{\mu(1-h)(1-\alpha)}{\mu(1-h)(1-\alpha)+(1-\mu)(1-\ell)(1-\alpha+\alpha y)}+1-\beta
$$

where $y$ is the lying probability of an $\ell$-type seller with reputation $\mu$. Other changes related to the effect of $\beta$ on prices and posteriors are detailed in the Online Appendix. 
The formula (21) shows that it is more difficult to penalise the seller for misreporting when $\alpha<1$ or $\beta<1$. Yet, it still shows that $\hat{\pi}_{G b}(\mu, y)$ goes to zero when $\alpha$ and $\beta$ tend to one unless the probability of lying goes to zero. Exploiting this property, together with the previous characterisation of an honest equilibrium for $\alpha=\beta=1$, we prove the existence of an honest equilibrium for large enough $\beta<1$ and $\alpha<1$, as stated below and proved in the Online Appendix. ${ }^{15}$ (Here, an honest equilibrium is one in which an $h$-type seller always announces his signal truthfully.)

Theorem 2 Given any $\ell \in(0,1)$, if $h$ and $\delta$ are sufficiently close to 1 then there exist $\underline{\beta}<1$ and $\underline{\alpha}(\beta)<1$ such that an honest equilibrium exists if $\beta \in(\underline{\beta}, 1)$ and $\alpha \in(\underline{\alpha}(\beta), 1)$. The value functions $V_{\theta}^{\beta \alpha}$ satisfy $\lim _{\beta \rightarrow 1} \lim _{\alpha \rightarrow 1} V_{\theta}^{\beta \alpha}=V_{\theta}^{*}$ uniformly on $(0,1)$ for $\theta \in\{h, \ell\}$.

The logic behind this result extends to other forms of imperfect monitoring. For instance, if the seller observes the quality perfectly but the buyers mis-report good quality as bad with probability $1-\alpha$, then an analogous result is obtained (see footnote 3 in the Online Appendix).

\subsection{Voluntary return policy}

The main insights of our analysis also apply when buyers can ask for a refund but the decision is left to the seller's discretion. Specifically, consider the following modification of the model: there is no pre-trade communication but the buyer observes quality ex-post and may return the good at no cost; when the good is returned, the seller observes quality and decides whether or not to reimburse the buyer; finally, the buyer publicly reports the quality $q$ and in case it is bad, whether the seller agreed to a refund $(B)$ or not $(G)$. In an honest equilibrium of this model, the item is sold for a price $p_{G}\left(\mu, y^{*}(\mu)\right) \in(0,1)$ when reputation is $\mu$, and an $h$-type seller always refunds bad quality items while an $\ell$-type seller refuses with probability $y^{*}(\mu)$.

In this equilibrium it is clearly optimal for the buyer to return the good if quality is bad and not if it is good. The buyer pays the price $p_{G}\left(\mu, y^{*}(\mu)\right)$ with probability $\mu h+(1-\mu)\left(\ell+(1-\ell) y^{*}(\mu)\right)$ only, and the expected utility from purchase is $\mu h+(1-\mu) \ell$. It thus follows that $p_{G}\left(\mu, y^{*}(\mu)\right)$ is indeed the equilibrium price.

Next, consider a seller's decision whether to reimburse or not. By reimbursing, the seller admits that the quality is bad and forgoes the short-term gain in exchange for future benefits from enhanced reputation. By refusing, the seller falsely claims the quality to be good for short-term gain but loses reputation afterward. As these choices are identical to those in our baseline model with pre-trade communication, the same equilibrium prevails.

The link between return policy and pre-trade communication has recently been investigated by Inderst and Ottaviani (2013) in a one-shot setting. In their model, commitment to a costly refund policy helps effective communication as the seller may prefer to avoid excessive returns. If the seller observes quality before trade in the model described above,

\footnotetext{
${ }^{15}$ The existence condition is more restrictive than the existence condition in Theorem 1 because we need to consider a value function $V_{h}^{*}$ that is continuous at $\mu=0$ as shown in the Online Appendix, while the equilibrium featured in Theorem 1 exhibits a value function discontinuous at $\mu=0$ for high ability seller.
} 
an honest equilibrium with pre-trade communication and one with refund policy are equivalent. However, the former is more efficient if the buyer or the seller bears a small return cost, which is in line with their analysis.

\subsection{Moral hazard and adverse selection}

To focus on the reputational incentives in pre-trade communication, we carried out our analysis in a model of pure adverse selection on seller's ability. However, the analysis can easily be extended to situations that involve moral hazard. In particular, modify the baseline model in such a way that in each period a seller draws an item of good quality with a probability $h$ if he exerted high effort at a cost of $c_{\theta}>0$ that depends on the seller's type $\theta \in\{h, \ell\}$; but he draws a good quality item with a probability $\ell$ if he exerted low effort at zero cost. For appropriate parameter values, our honest equilibrium continues to be an equilibrium in this modified model if $c_{h}$ is small enough for an $h$-type seller to find it worthwhile to exert high effort, but $c_{\ell}$ is so large that an $\ell$-type seller finds it undesirable.

This is the case when the following holds for all $\mu \geq \mu_{1}$ :

$$
\begin{aligned}
\delta\left(V_{h}^{*}\left(\pi_{B b}\left(\mu, y^{*}(\mu)\right)-\frac{c_{h}}{1-\delta}-\frac{\ell}{1-\delta}\right)\right. & \geq p_{G}\left(\mu, y^{*}(\mu)\right), \\
(h-\ell)\left(p_{G}\left(\mu, y^{*}(\mu)\right)+\delta V_{h}^{*}\left(\pi_{G g}^{*}(\mu)\right)-\delta V_{h}^{*}\left(\pi_{B b}\left(\mu, y^{*}(\mu)\right)\right)\right) & \geq c_{h}, \\
\text { and }(h-\ell) \delta\left(V_{\ell}^{*}\left(\pi_{G g}^{*}(\mu)\right)-\frac{\ell}{1-\delta}\right) & \leq c_{\ell},
\end{aligned}
$$

where $y^{*}, V_{\ell}^{*}$ and $V_{h}^{*}$ are as derived in Section 4 . The first inequality is the condition that an $h$-type seller prefers to tell the truth upon drawing a bad quality, the second that an $h$-type seller prefers to exert effort to not, and the third that an $\ell$-type seller prefers to not exert effort. Values of $c_{h}$ and $c_{\ell}$ that satisfy these conditions exist for $\mu_{1}$ not too small because $p_{G}\left(\mu, y^{*}(\mu)\right)>\ell$ and $V_{h}^{*}\left(\pi_{G g}^{*}(\mu)\right)-V_{h}^{*}\left(\pi_{B b}\left(\mu, y^{*}(\mu)\right)\right)$ vanishes as $\mu$ tends to 1 .

If pre-trade communication is not possible, this model is equivalent to the baseline model of Mailath and Samuelson (2001) without replacement of types, for which they show that high effort cannot be induced unless discontinuous strategies are allowed (Proposition 2, p. 424). Our result shows that pre-trade communication may motivate the more efficient type to exert high effort by facilitating learning. It is then straightforward that welfare is higher in the equilibrium with communication than in the equilibrium without.

\subsection{Negative social value and no trading of bad quality}

In our baseline model trade takes place efficiently in every period because myopic buyers are assumed to value the good more than the seller for sure. This feature precludes a simple solution of resorting to a trigger strategy to discipline a seller. If buyers value a bad quality item less than the seller's cost of providing it, on the other hand, low quality items would not be traded in an honest equilibrium whereas they would still be traded in the babbling equilibrium due to lack of information transmission. In this case, communication enhances social welfare by preventing inefficient trade. 
To elaborate on this idea, suppose that a seller incurs a small distribution cost $c<$ $\ell$ for each sale. Then, our equilibrium characterisation continues to be valid with the modification that the seller's income is $p_{G}(\mu, y)-c$ when he announces $G$ while no trade occurs when he announces $B$. After a lie, a seller's reputation collapses to nil and the seller's continuation value is $(\ell-c) /(1-\delta)$. The condition for an $h$-type seller to truthfully announce a bad quality gets relaxed because it avoids paying the cost $c$, which is reflected in a lower value of $\delta_{h}=(1-c) /(1+h(1-c)-\ell)$. However, additional incentive constraints need to be accounted for due to the following fact: as quality is not observed by the market when $B$ is announced, a seller with a good quality item has an option of raising his reputation to $\pi_{B b}^{*}(\mu)$ by announcing $B$. It is easy to verify that an $\ell$-type seller has no incentive to do this because he would opt for a short-term gain, $p_{G}^{*}(\mu)-c$, over even a larger potential reputation gain in the case that a bad quality item is drawn. Yet, an $h$-type seller may be tempted. We show in the Online Appendix that this is not the case and that an honest equilibrium exists for $\delta>\delta_{h}$ when the initial reputation is not too small.

Note that in this extension, communication may arise even if the seller's type is common knowledge if we allow for non-Markov strategies. In a simple such equilibrium, the seller sells only good quality items and the buyers believe the quality of items for sale to be good as long as it has been in the past, and to be an average otherwise. The seller's equilibrium payoff is $\theta(1-c) /(1-\delta)$ which is larger than the deviation payoff $1+\delta(\theta-c) /(1-\delta)$ if the seller is patient enough, and the equilibrium is efficient. Our baseline model abstracts from this possibility to focus on incentives to announce quality in a more basic environment.

\section{$7 \quad$ New-life}

Up to now we have assumed that sellers stay in the marketplace forever and that memory is infinite. One of the key issues surrounding the reputation mechanisms based on feedback systems is that sellers may find ways to escape from the bad consequences of damaged reputation. For instance, sellers may change to another marketplace, or they may change their identities within the same marketplace and start again as a new seller. ${ }^{16}$ One may then be concerned that such a possibility may destroy the fundamental reputation mechanism delineated in Section 4. We show that this is not the case, although the effectiveness of the mechanism in fostering honest communication by the seller is somewhat reduced.

To address this issue, we need to introduce inflow and outflow of sellers in each period. Specifically, we consider a marketplace or website with a stationary mass 1 of sellers, where a small fraction $\chi \in(0,1)$ of randomly chosen sellers "die" at the end of each period and a new cohort of "newborn" sellers of mass $\chi$ enters the marketplace. Each new born seller is of $h$-type with an exogenously given probability $\gamma \in(0,1)$. Thus, there are many sellers in the market but we assume that they do not compete either because their goods are

\footnotetext{
${ }^{16}$ The ability to do so depends on the technology used by the platforms. This is known to be an issue with eBay for instance (Delarocas 2006), but would be less of an issue when the platform controls bank coordinates or social status of companies, for then it would involve creating a new firm which is costly.
} 
non-rival or because there are more buyers than sellers. ${ }^{17}$

Note that in this setting, discounting must reflect the survival rate. We define $\delta$ as the seller's discount factor adjusted for survival, i.e., the discount factor multiplied by the survival rate $1-\chi$.

We maintain the assumptions that there is no credible outside option for a seller and that the past record of each seller is publicly known. But we now allow sellers to restart as a new seller and buyers cannot distinguish a newborn seller from an old seller restarting with a clean record. We then focus on stationary equilibria satisfying Condition $\mathrm{H}$, such that the proportion of $\ell$-type sellers restarting afresh is constant every period, while $h$-type sellers never lie and consequently, never change their identities.

In a stationary equilibrium, a constant mass, denoted by $\chi_{1}$, of new sellers (newborns and restarters) appear on the platform in each period and they start with an endogenously determined "default reputation level," denoted by $\mu_{1}$, which reflects the mix of genuine newborn sellers (of which a proportion $\gamma$ are of $h$-type) and equilibrium mass of sellers who restart. Let $v_{1}$ denote the equilibrium value of an $\ell$-type seller starting at the default reputation level $\mu_{1}$. This must be larger than the minimum value when restarting is not possible, i.e., $v_{1}>\ell /(1-\delta)$. Notice that the levels of $v_{1}$ and $\mu_{1}$ are determined endogenously, together with the equilibrium announcement strategy and value functions. We provide a sketch of how they are determined below, while deferring a formal analysis to the Online Appendix.

In the honest equilibrium studied in Section 4 , the analysis remains intact if the seller is assumed to exit the market with an exit value of $V_{\theta}^{*}(0)=\ell /(1-\delta)$ once his reputation falls to 0 due to a false claim of good quality. As an intermediate step toward the current model with restarting possibilities, we may consider an extension of the baseline model without restarting but treating $v_{1}>\ell /(1-\delta)$ as an exogenous exit value that sellers get by exiting the market when their reputation falls to 0 . One can establish that, for any given $v_{1}$, an analogous honest equilibrium exists by the same arguments as in Section 4 . The seller's strategy in the equilibrium with restart possibilities must then coincide with that in this equilibrium, except that a seller restarts when he would exit. Given that an $h$-type seller's value surely increases in every period in the honest equilibrium with exit, this establishes in particular that an $h$-type seller never restarts even if it is allowed, as postulated above. The price dynamics for an $h$-type seller is thus the same as in the base model. But, an $\ell$-type seller restarts on the first date after he lied and revealed his type. ${ }^{18}$

Let $y_{v_{1}}^{\dagger}(\mu)$ and $V_{v_{1}}^{\dagger}(\mu)$ denote the equilibrium lying probability (when $q=b$ ) and value function of an $\ell$-type seller in the honest equilibrium with exit, where the subscript $v_{1}$ indicates their dependence on the exit value $v_{1}$. In this equilibrium, one can obtain the ex-ante probability that a new $\ell$-type seller will exit (but not die) at some future period, denoted by $\Lambda\left(v_{1}, \mu_{1}\right)$, by adding up the ex-ante probabilities that he will lie for the first

\footnotetext{
${ }^{17}$ As a result, all sellers trade and a mechanism that would rely on exclusion and replacement of misbehaving sellers, as in Inderst and Ottaviani (2012), would not be credible.

${ }^{18}$ Atkeson, Hellwig and Ordonez (2012) and Board and Meyer-Ter-Vehn (2013) show that the dynamics depends on whether learning occurs through good news or through bad news. In our model, the dynamics resembles the bad news case in that reputation increases until a false announcement of $G$ reveals a low type.
} 
time in period $k=1,2, \cdots$, according to $y_{v_{1}}^{\dagger}$ when the seller's reputation evolves by Bayes' rule (based on $y_{v_{1}}^{\dagger}$ ) from an initial reputation $\mu_{1}$. In an honest equilibrium with restart possibilities, $\Lambda\left(v_{1}, \mu_{1}\right)$ would be the ex-ante probability that a new $\ell$-type seller will restart at some future period, which will determine the equilibrium default reputation level and thereby, the equilibrium value of a newly starting $\ell$-type seller. If these values coincide respectively with the values of $\mu_{1}$ and $v_{1}$ that we began with, we have identified an honest equilibrium with restart possibilities. This process is elaborated below.

As the mass of newly starting $\ell$-type sellers is $\chi_{1}\left(1-\mu_{1}\right)$ in each period of such an equilibrium, the mass of sellers who change their identities in a stationary equilibrium is $\chi_{1}\left(1-\mu_{1}\right) \Lambda\left(v_{1}, \mu_{1}\right)$ where $v_{1}$ is the value of a newly starting $\ell$-type seller. Stationarity then dictates the mass of new sellers to be

$$
\chi_{1}=\chi+\chi_{1}\left(1-\mu_{1}\right) \Lambda\left(v_{1}, \mu_{1}\right) .
$$

As only $\ell$-type sellers restart, Bayes' rule dictates that in a stationary state

$$
\mu_{1}=\frac{\gamma \chi}{\chi_{1}}
$$

Solving (22) and (23) simultaneously we set the initial reputation $\mu_{1}$ as the solution of

$$
\mu_{1}=\frac{\gamma-\gamma \Lambda\left(v_{1}, \mu_{1}\right)}{1-\gamma \Lambda\left(v_{1}, \mu_{1}\right)}<\gamma
$$

where the inequality follows from $0<\Lambda\left(v_{1}, \mu_{1}\right)$. This characterizes the default reputation level of new sellers, that is consistent with a given value $v_{1}$ of a newly starting $\ell$-type seller. Finally, the equation $v_{1}=V_{v_{1}}^{\dagger}\left(\mu_{1}\right)$ must hold in a stationary equilibrium. By solving the system of equation for $v_{1}$ and $\mu_{1}$, we establish (in the Online Appendix) that

Theorem 3 If sellers can change their identity costlessly in any period, there exists a stationary honest equilibrium if $h$ and $\delta$ are sufficienty large and $\gamma$ is not too large. In this equilibrium the probability with which an $\ell$-type seller lies when $q=b$ exceeds $y^{*}(\mu)$ obtained in Section 4 for all relevant reputation levels.

The main conclusion is thus that allowing sellers to change identity at no cost doesn't invalidate our result that equilibria exist in which sellers of high ability always communicate truthfully. Unsurprisingly, however, it impedes the power of incentives somewhat. In particular, the benefit of keeping reputation is lower than when identities cannot be changed. As a result, an $\ell$-type seller lies more.

Interestingly, this does not mean that untruthful announcements are more frequent when restarts are possible than when they are not, because $\ell$-type sellers who have lied once, rather than keep lying forever when $q=b$, would start afresh and announce according to the equilibrium strategy. In fact, when $\delta$ is close to 1 there will be more truthful announcements in the market when sellers are allowed to restart with a new identity.

The reduced reliability of $\ell$-type sellers results in lower prices for all reputation levels, but the separation process takes place faster due to an enlarged gap in the honesty levels of the two types. On the other hand, newborn sellers suffer from depressed reputation at birth due to the restarters who are all $\ell$-type. Therefore, it is unclear whether it takes longer or not for an $h$-type seller to be identified as such by the market. 


\section{Concluding remarks}

Our model highlights the role of reputation in establishing the market's trust in a seller's communication. When the seller's ability is common knowledge, the unique equilibrium price is constant and equal to the expected quality. Hence, asymmetric information on ability is essential for credible communication to take place. Our analysis emphasises the critical role of both the information structure and the communication system for trading platforms and marketplaces, and the benefits of monitoring the quality of information provided by traders.

We show that in repeated environments the anticipation of future communication is sufficient to generate separation of types and that truthful announcement of quality by high ability sellers is self-supporting. As a result, pre-trade communication helps mitigate the asymmetric information problem along two interrelated dimensions: $i$ ) it enables credible communication of the item's quality, and $i i$ ) it facilitates consumers' learning of the seller's ability.

As explained in the Introduction, these effects are possible because a seller's history (the feedback comments) is utilized to infer the accuracy of pre-trade communication as well as the quality delivered. Imperfection of the signal or the ability to restart with a new identity reduce, but do not undermine effective transmission of information, provided that sellers are patient enough. Various extensions are also discussed where our results imply that communication has a positive welfare effect.

We anticipate that our analysis can be extended in other directions as well: for instance, in the context of Internet markets, examining the effect of competition between trading websites appears to be an interesting task from the market design perspective, particularly because rival websites would influence each other by providing exit values as our results suggest. Analysis of such competition may also carry implications for the market segmentation between trading websites and their pricing strategies. At the same time, it also seems fruitful to explore other routes to enhance the value of online reputation: for instance, via creating a market for trading online identities a la Tadelis (1999).

\section{A Appendix to Section 4}

\section{A.1 Justification of a binary message space $M=\{G, B\}$}

Lemma 3 Consider an arbitrary honest equilibrium represented by $s^{*}, p^{*}$ and $\pi^{*}$. In a period with any given reputation $\mu \in(0,1]$, let $M_{g}$ and $M_{b}$ denote the sets of messages sent by an h-type seller with a positive probability when $q=g$ and $q=b$, respectively.

(a) If $s_{\mu}^{*}(\ell, g)(m)>0$, then $m \in M_{g}$;

(b) If $s_{\mu}^{*}(\ell, b)(m)>0$, then $m \in M_{g} \cup M_{b}$;

(c) If $m, m^{\prime} \in M_{q}$ for some $q \in\{g, b\}$, then i) $p_{m}^{*}(\mu)=p_{m^{\prime}}^{*}(\mu)$ and $\pi_{m q}^{*}(\mu)=\pi_{m^{\prime} q}^{*}(\mu)$ and ii) $\pi_{m b}^{*}(\mu)=\pi_{m^{\prime} b}^{*}(\mu)=0$ if $q=g$ and $\mu<1$.

A proof is provided below. By (a) and (b) of Lemma 3, in any honest equilibrium it is innocuous to restrict the message space to only those used by an $h$-type seller. By (c), for 
any $\mu \in(0,1)$, any two messages that an $h$-type seller sends with positive probabilities for a given quality induce the same price and the same posterior beliefs in the next period, except possibly in some off-equilibrium contingencies. Hence, the equilibrium conditions continue to hold when the off-equilibrium specifications of one of the two messages are replaced by those of the other message. This means that it is innocuous to identify all messages that an $h$-type seller sends when $q=g$ as a single message $G$, and all messages that he sends when $q=b$ as a single message $B$. When $\mu=1$, part (c) holds except that $\pi_{m b}^{*}(\mu)$ and $\pi_{m^{\prime} b}^{*}(\mu)$ are not defined by Bayes' rule when $q=g$. But, setting $\pi_{m^{\prime} b}^{*}(\mu)$ equal to $\pi_{m b}^{*}(\mu)$ in this case does not affect equilibrium conditions, so the same conclusion holds. This justifies postulating $M=\{G, B\}$ in any honest equilibrium.

Proof of Lemma 3. The argument in Section 4.1 leading to $V_{\ell}^{*}(0)=\ell /(1-\delta)$ is valid for an arbitrary message space and hence, it holds for the honest equilibrium considered here.

For any $\mu>0$, there is a message $m$ with $p_{m}^{*}(\mu)>\ell$ because the expected value of the prices over all messages is equal to the expected quality given $\mu, \mathbb{E}(q \mid \mu)>\ell$. This means that the seller can get a price strictly greater than $\ell$ in any period with reputation $\mu>0$ and a price no lower than $\ell$ in any future period. Therefore,

$$
V_{\ell}^{*}(0)=\frac{\ell}{1-\delta}<V_{\ell}^{*}(\mu) \quad \forall \mu>0 .
$$

For any given $\mu>0$, consider an $\ell$-type seller with a good quality item. If he were to announce a message $m \notin M_{g}$ with a positive probability, then $p_{m}^{*}(\mu)$ must be the highest among all messages and strictly higher than $p_{G}^{*}(\mu)$ for any $G \in M_{g}$ because $\pi_{m g}^{*}(\mu)=0$. Then, an $\ell$-seller does not announce $G$ when $q=b$ with a positive probability (because announcing $m$ is better), either, which implies that $p_{G}^{*}(\mu)=1$, contradicting the earlier assertion that $p_{m}^{*}(\mu)>p_{G}^{*}(\mu)$. Thus, an $\ell$-type seller with a good quality item must not announce any $m \notin M_{g}$. This proves part (a).

Given this, if an $\ell$-type seller with a low quality item were to announce a message $m \notin M_{g} \cup M_{b}$, then it would follow that $p_{m}^{*}(\mu)=0$ and $\pi_{m B}^{*}(\mu)=0$, contradicting the presumed optimality of announcing $m$ because announcing any message in $M_{g}$ is better. This proves part (b).

By (a) and (b), we have $p_{G}^{*}(\mu)>p_{B}^{*}(\mu)=0$ for all $\mu>0$ if $G \in M_{g}$ and $B \in M_{b}$.

Suppose that $B, B^{\prime} \in M_{b}$. Then, $p_{B}^{*}(\mu)=p_{B^{\prime}}^{*}(\mu)=0$ as shown above. In addition, to show that $\pi_{B b}^{*}(\mu)=\pi_{B^{\prime} b}^{*}(\mu)>0$ by contradiction, suppose otherwise, say $0<\pi_{B b}^{*}(\mu)<$ $\pi_{B^{\prime} b}^{*}(\mu)$. Note that $V_{h}^{*}\left(\pi_{B b}^{*}(\mu)\right)=V_{h}^{*}\left(\pi_{B^{\prime} b}^{*}(\mu)\right)$ should hold for an $h$-seller to send both $B$ and $B^{\prime}$. Since it is also optimal for an $\ell$-seller to send $B$ when $q=b$ (because $0<\pi_{B b}^{*}(\mu)<$ 1), $V_{\ell}^{*}\left(\pi_{B b}^{*}(\mu)\right) \geq V_{\ell}^{*}\left(\pi_{B^{\prime} b}^{*}(\mu)\right)$ should hold. At the same time,

$$
\begin{aligned}
\pi_{B b}^{*}(\mu) V_{h}^{*}\left(\pi_{B b}^{*}(\mu)\right)+\left(1-\pi_{B b}^{*}(\mu)\right) V_{\ell}^{*}\left(\pi_{B b}^{*}(\mu)\right) & =\mathbb{E}\left(q \mid \pi_{B b}^{*}(\mu)\right) /(1-\delta) \text { and } \\
\pi_{B^{\prime} b}^{*}(\mu) V_{h}^{*}\left(\pi_{B^{\prime} b}^{*}(\mu)\right)+\left(1-\pi_{B^{\prime} b}^{*}(\mu)\right) V_{\ell}^{*}\left(\pi_{B^{\prime} b}^{*}(\mu)\right) & =\mathbb{E}\left(q \mid \pi_{B^{\prime} b}^{*}(\mu)\right) /(1-\delta)
\end{aligned}
$$

must hold. Replacing $V_{\theta}^{*}\left(\pi_{B^{\prime} b}^{*}(\mu)\right)$ with $V_{\theta}^{*}\left(\pi_{B b}^{*}(\mu)\right)$ for $\theta=h, \ell$ on the left hand side of the second equation, then subtracting the two equations side by side and rearranging, we get $V_{h}^{*}\left(\pi_{B b}^{*}(\mu)\right)-V_{\ell}^{*}\left(\pi_{B b}^{*}(\mu)\right) \geq(h-\ell) /(1-\delta)$, which is impossible due to (25) because $p_{B}^{*}(\mu)=0$ and $p_{G}^{*}(\mu) \leq 1$. This proves $\pi_{B b}^{*}(\mu)=\pi_{B^{\prime} b}^{*}(\mu)$ and thereby, part (c) when $q=b$. 
Next, suppose that $G, G^{\prime} \in M_{g}$. If $p_{G}^{*}(\mu)=p_{G^{\prime}}^{*}(\mu)$, then $\pi_{G g}^{*}(\mu)=\pi_{G^{\prime} g}^{*}(\mu)$ must also hold by an argument analogous to the above. Consider the alternative case, say $0<p_{G}^{*}(\mu)<p_{G^{\prime}}^{*}(\mu)$. Then, $V_{h}^{*}\left(\pi_{G g}^{*}(\mu)\right)-V_{h}^{*}\left(\pi_{G^{\prime} g}^{*}(\mu)\right)=p_{G^{\prime}}^{*}(\mu)-p_{G}^{*}(\mu) \leq V_{\ell}^{*}\left(\pi_{G g}^{*}(\mu)\right)-$ $V_{\ell}^{*}\left(\pi_{G^{\prime} g}^{*}(\mu)\right)$ where the equality follows because $G$ and $G^{\prime}$ are equivalent for an $h$-seller when $q=g$ and the inequality because $G$ is optimal for an $\ell$-seller to send when $q=g$. Then, applying analogous calculations to

$$
\begin{aligned}
\pi_{G g}^{*}(\mu) V_{h}^{*}\left(\pi_{G g}^{*}(\mu)\right)+\left(1-\pi_{G g}^{*}(\mu)\right) V_{\ell}^{*}\left(\pi_{G g}^{*}(\mu)\right) & =\mathbb{E}\left(q \mid \pi_{G g}^{*}(\mu)\right) /(1-\delta) \text { and } \\
\pi_{G^{\prime} g}^{*}(\mu) V_{h}^{*}\left(\pi_{G^{\prime} g}^{*}(\mu)\right)+\left(1-\pi_{G^{\prime} g}^{*}(\mu)\right) V_{\ell}^{*}\left(\pi_{G^{\prime} g}^{*}(\mu)\right) & =\mathbb{E}\left(q \mid \pi_{G^{\prime} g}^{*}(\mu)\right) /(1-\delta),
\end{aligned}
$$

we deduce $V_{h}^{*}\left(\pi_{G g}^{*}(\mu)\right)-V_{\ell}^{*}\left(\pi_{G g}^{*}(\mu)\right) \geq(h-\ell) /(1-\delta)$, which is impossible as above. Note that $\pi_{G b}^{*}(\mu)=\pi_{G^{\prime} b}^{*}(\mu)=0$ for $\mu<1$ by Bayes' rule. This proves part (c) when $q=g$.

\section{A.2 Justification of the off-equilibrium beliefs in (12)}

From now on, we take it for granted that $M=\{G, B\}$ where $G$ and $B$ are the messages sent by an $h$-type seller when $q=g$ and $q=b$, respectively, as stipulated due to Lemma 3 .

We show that our specification of off-equilibrium beliefs as in (12) is innocuous as it does not affect optimal strategy on the equilibrium path. As a seller always tells the truth when $q=g$ in an honest equilibrium due to Condition $\mathrm{H}$ and Lemma $3(\mathrm{a}), \pi_{B g}^{*}(\mu) \equiv 0$ maximises such incentives and does not affect any other equilibrium conditions.

Setting $\pi_{G b}^{*}(1)=0$ is innocuous for an $h$-type seller by the same reason. But, verifying it for an $\ell$-type seller needs to be done more carefully as he is supposed to announce $G$ when $\mu=1$ and $q=b$. First, observe that if $y^{*}(1)<1$ then telling the truth would be optimal when $\mu=1$ and $q=b$, so we would have

$$
V_{\ell}^{*}(1)=\ell\left(1+\delta V_{\ell}^{*}(1)\right)+(1-\ell) \delta V_{\ell}^{*}(1)=\ell+\delta V_{\ell}^{*}(1) \Rightarrow V_{\ell}^{*}(1) \leq \ell /(1-\delta)=V_{\ell}^{*}(0),
$$

contradicting (25). Hence, we deduce that $y^{*}(1)=1$ and therefore, $V_{\ell}^{*}(1)=1+\delta V_{\ell}^{*}\left(\pi_{G b}^{*}(1)\right)$.

Next, suppose that $\pi_{G b}^{*}(1)>0$ in an honest equilibrium. If we reset $\pi_{G b}^{*}(1)=0$ without changing $y^{*}(\cdot)$, the value $V_{\ell}^{*}(1)$ is lower (weakly) and equal to $1+\delta V_{\ell}^{*}(0)$, while $V_{\ell}^{*}(\mu)$ is unchanged for $\mu<1$ (because $V_{\ell}^{*}(1)$ does not affect $V_{\ell}^{*}(\mu)$ for $\mu<1$ ). Hence, the incentive compatibility for an $\ell$-type seller is preserved for $\mu<1$. It is preserved for $\mu=1$ as well because $1+\delta V_{\ell}^{*}(0)>\delta\left(1+\delta V_{\ell}^{*}(0)\right)=\delta V_{\ell}^{*}(1)$. This establishes that we may set $\pi_{G b}^{*}(1)=0$ without affecting optimal strategy on the equilibrium path.

Lastly, as an $\ell$-type seller's reputation will remain at 0 forever once it reaches 0 , setting $\pi_{B b}^{*}(0)=0$ does not affect $\ell$-type seller's incentives. For an $h$-type seller, $\pi_{B b}^{*}(0)=0$ minimises his equilibrium value at $\mu=0, V_{h}^{*}(0)$, by keeping him trapped at $\mu=0$ once he reaches there. As this maximises his incentive to tell the truth when $\mu>0$ by minimising his value after spoiling reputation by lying, it does not affect his equilibrium behaviour of truth-telling when $\mu>0$. When $\mu=0$, he lies when $q=b$ but this does not affect the equilibrium path because $\mu=0$ is an off-equilibrium contingency for an $h$-type seller. 


\section{A.3 Proof of Lemma 2 and some intermediate results}

Recall that we deduced $y^{*}(1)=1$ and $\Delta=V_{\ell}^{*}(1)-V_{\ell}^{*}(0)<1$ in (13). We start by showing

$$
V_{\ell}^{*}(\mu) \leq V_{\ell}^{*}(1) \quad \forall \mu<1 .
$$

First, note that $V_{\ell}^{*}(1)=\frac{1+\delta(1-\ell) V_{\ell}^{*}(0)}{1-\delta \ell}$. To reach a contradiction, suppose $V_{\ell}^{*}(\mu)>V_{\ell}^{*}(1)$ for some $\mu<1$ in an honest equilibrium. Then, one can find arbitrary small $\epsilon>0$ and $\mu_{\epsilon}<1$ such that

$$
V_{\ell}^{*}\left(\mu_{\epsilon}\right)>\sup _{\mu<1} V_{\ell}^{*}(\mu)-\epsilon>V_{\ell}^{*}(1)+\frac{\delta}{1-\delta \ell} \epsilon>V_{\ell}^{*}(0)+\frac{\delta}{1-\delta} \epsilon .
$$

If $y^{*}\left(\mu_{\epsilon}\right)>0$, then $V_{\ell}^{*}\left(\mu_{\epsilon}\right)=p_{G}^{*}\left(\mu_{\epsilon}\right)+\delta\left(\ell V_{\ell}^{*}\left(\pi_{G g}^{*}\left(\mu_{\epsilon}\right)\right)+(1-\ell) V_{\ell}^{*}(0)\right)<1+\delta\left(\ell\left(V_{\ell}^{*}\left(\mu_{\epsilon}\right)+\right.\right.$ $\left.\epsilon)+(1-\ell) V_{\ell}^{*}(0)\right) \Rightarrow V_{\ell}^{*}\left(\mu_{\epsilon}\right)<\frac{1+\delta(1-\ell) V_{\ell}^{*}(0)}{1-\delta \ell}+\frac{\delta}{1-\delta \ell} \epsilon=V_{\ell}^{*}(1)+\frac{\delta}{1-\delta \ell} \epsilon$, violating (27). If $y^{*}\left(\mu_{\epsilon}\right)=0$, then $V_{\ell}^{*}\left(\mu_{\epsilon}\right)=\ell p_{G}^{*}\left(\mu_{\epsilon}\right)+\delta\left(\ell V_{\ell}^{*}\left(\pi_{G g}^{*}\left(\mu_{\epsilon}\right)\right)+(1-\ell) V_{\ell}^{*}\left(\pi_{B b}^{*}\left(\mu_{\epsilon}\right)\right)\right) \leq \ell+$ $\delta \sup _{\mu<1} V_{\ell}^{*}(\mu)<\ell+\delta\left(V_{\ell}^{*}\left(\mu_{\epsilon}\right)+\epsilon\right) \Rightarrow V_{\ell}^{*}\left(\mu_{\epsilon}\right)<V_{\ell}^{*}(0)+\frac{\delta}{1-\delta} \epsilon$, contradicting (27). Hence, (26) is proved.

Next, we show that

$$
y^{*}(\mu)>0 \quad \forall \mu \in[0,1]
$$

This is trivial for $\mu=0$ by our convention that $y^{*}(0)=1$. For $\mu \in(0,1), y^{*}(\mu)=0$ would imply that the short-term gain from lying when $q=b$, which is $p_{G}^{*}(\mu)-p_{B}^{*}(\mu)=1$, would exceed the long-term loss, $\delta\left(V_{\ell}^{*}\left(\pi_{B b}^{*}(\mu)\right)-V_{\ell}^{*}\left(\pi_{G b}^{*}(\mu)\right)\right)<\delta \Delta$ where the inequality follows from (26) and (25). This contradicts the optimality of $y^{*}(\mu)=0$, establishing (28).

Hence, any equilibrium value function $V_{\ell}^{*}$ should satisfy

$$
V_{\ell}^{*}(\mu)=p_{G}\left(\mu, y^{*}(\mu)\right)+\delta\left(\ell V_{\ell}^{*}\left(\pi_{G g}^{*}(\mu)\right)+(1-\ell) V_{\ell}^{*}(0)\right) \quad \forall \mu \in[0,1] .
$$

Let $\pi_{G g}^{1}(\mu)=\pi_{G g}^{*}(\mu)$ and $\pi_{G g}^{t}(\mu)=\pi_{G g}^{*}\left(\pi_{G g}^{t-1}(\mu)\right)$ recursively for $t \geq 2$ so that

$$
\pi_{G g}^{t}(\mu)=\frac{\mu h^{t}}{\mu h^{t}+(1-\mu) \ell^{t}}>\mu \text {. }
$$

Then, expanding (29) by applying an analogous equation to $V_{\ell}^{*}\left(\pi_{G g}^{*}(\mu)\right)$ repeatedly,

$$
\begin{aligned}
V_{\ell}^{*}(\mu) & =\left[\sum_{t=0}^{\infty} \ell^{t} \delta^{t} p_{G}\left(\pi_{G g}^{t}(\mu), y^{*}\left(\pi_{G g}^{t}(\mu)\right)\right)\right]+\delta V_{\ell}^{*}(0)(1-\ell) \sum_{t=0}^{\infty} \ell^{t} \delta^{t} \\
& =\sum_{t=0}^{\infty} \delta^{t} \ell^{t}\left(p_{G}\left(\pi_{G g}^{t}(\mu), y^{*}\left(\pi_{G g}^{t}(\mu)\right)\right)-\ell\right)+V_{\ell}^{*}(0)
\end{aligned}
$$

Now we derive three intermediate results that will be combined to prove Lemma 2.

Lemma 4 For any honest equilibrium, there exists $\bar{\mu}<1$ (defined in (8)) such that $y^{*}(\mu)=1$ if $\mu>\bar{\mu}$. Furthermore, $V_{\ell}^{*}(\mu)$ is continuous and strictly increasing on $(\bar{\mu}, 1]$. 
Proof. As the short-term gain from lying when $q=b, p_{G}(\mu, y)$, is decreasing in $y$ while the long-term loss is bounded above by $\delta \Delta$, we deduce that $y^{*}(\mu)=1$ so long as $p_{G}(\mu, 1) \geq \delta \Delta$, i.e., for all $\mu \geq \bar{\mu}$ where $\bar{\mu}$ is defined in (8). Then, since $\left.y^{*}\left(\pi_{G g}^{t}(\mu)\right)\right)=1$ for all $t$ for any $\mu \geq \bar{\mu}$ by (30), (31) uniquely determines $V_{\ell}^{*}(\mu)$ for $\mu \geq \bar{\mu}$ as

$$
V_{\ell}^{*}(\mu)=\left[\sum_{t=0}^{\infty} \ell^{t} \delta^{t} p_{G}\left(\pi_{G g}^{t}(\mu), 1\right)\right]+\delta V_{\ell}^{*}(0)(1-\ell) \sum_{t=0}^{\infty} \ell^{t} \delta^{t}
$$

which is continuous and strictly increasing in $\mu>\bar{\mu}$ because both $p_{G}(\mu, 1)$ and $\pi_{G g}^{*}(\mu)$ are continuous and strictly increasing in $\mu$. Moreover, $\lim _{\mu \rightarrow 1} V_{\ell}^{*}(\mu)=V_{\ell}^{*}(1)$, verifying continuity at $\mu=1$.

Lemma 5 In any honest equilibrium, if $y^{*}(\mu)$ is continuous on an interval $\left(\mu_{0}, 1\right]$ then $\pi_{B b}\left(\mu, y^{*}(\mu)\right)>\mu$ for all $\mu \in\left(\mu_{0}, 1\right)$, and $p_{G}^{*}(\mu)$ and $V_{\ell}^{*}(\mu)$ are continuous and strictly increasing in $\mu \in\left(\mu_{0}, 1\right]$.

Proof. The proof is immediate if $\mu_{0} \geq \bar{\mu}$ by Lemma 4 . Hence, we consider the case that $\mu_{0}<\bar{\mu}$ below. Let $\mu^{\prime} \in\left(\mu_{0}, \bar{\mu}\right]$ be such that $V_{\ell}^{*}(\mu)$ is continuous and strictly increasing in $\mu \in\left(\mu^{\prime}, 1\right]$. It exists by Lemma 4 . We proceed by showing that the properties in Lemma 5 hold on $\left(\mu^{\prime}-\varepsilon, 1\right]$ for small enough $\varepsilon>0$, hence on $\left(\mu_{0}, 1\right]$, in three steps as below.

Step 1: First, we verify that

$$
\pi_{B b}\left(\mu, y^{*}(\mu)\right)>\mu \quad \forall \mu \in\left[\mu^{\prime}, 1\right) .
$$

To reach a contradiction, suppose to the contrary that there is some $\mu \in\left[\mu^{\prime}, 1\right)$ such that $\pi_{B b}\left(\mu, y^{*}(\mu)\right) \leq \mu$. Then, by continuity of $\pi_{B b}(\mu, y)$ and $y^{*}(\mu)$ on $\left(\mu_{0}, 1\right]$, we can define

$$
\tilde{\mu}=\max \left\{\mu<1 \mid \pi_{B b}\left(\mu, y^{*}(\mu)\right) \leq \mu\right\} \in\left[\mu^{\prime}, \bar{\mu}\right) .
$$

Here, $\tilde{\mu} \in\left[\mu^{\prime}, \bar{\mu}\right)$ follows because for $\mu \in[\bar{\mu}, 1)$, by Lemma $4, y^{*}(\mu)=1$ and thus, $\pi_{B b}\left(\mu, y^{*}(\mu)\right)=1>\mu$. By continuity, we also have $\pi_{B b}\left(\tilde{\mu}, y^{*}(\tilde{\mu})\right)=\tilde{\mu}$.

It is easily verified from (5) that

$$
\pi_{B b}(\mu, y) \geq \mu \Longleftrightarrow y \geq \hat{y}=\frac{h-\ell}{1-\ell},
$$

which implies that $y^{*}(\tilde{\mu})=\hat{y}$ and thus

$$
p_{G}(\tilde{\mu}, \hat{y})=\delta\left(V_{\ell}^{*}(\tilde{\mu})-V_{\ell}^{*}(0)\right) \text {. }
$$

Note from (30) that $\pi_{G g}^{t}(\tilde{\mu})>\tilde{\mu}$ for $t \geq 1$ and thus, $\pi_{B b}\left(\pi_{G g}^{t}(\tilde{\mu}), y^{*}\left(\pi_{G g}^{t}(\tilde{\mu})\right)\right)>\pi_{G g}^{t}(\tilde{\mu})$ by (34). Consequently, $y^{*}\left(\pi_{G g}^{t}(\tilde{\mu})\right)>\hat{y}$ by (35). Therefore, since $p_{G}(\mu, y) \leq 1$ and $p_{G}(\mu, y)$ decreases in $y,(32)$ implies that

$$
V_{\ell}^{*}(\tilde{\mu})-V_{\ell}^{*}(0)<\sum_{t=0}^{\infty}\left(p_{G}\left(\pi_{G g}^{t}(\tilde{\mu}), \hat{y}\right)-\ell\right) \delta^{t} \ell^{t}
$$


Since $p_{G}(\mu, \hat{y})=(\mu(h-\ell)+\ell) / h$ from (4) and (35), we further deduce from (37) that

$$
\begin{aligned}
V_{\ell}^{*}(\tilde{\mu})-V_{\ell}^{*}(0) & <p_{G}(\tilde{\mu}, \hat{y})-\ell+\sum_{t=1}^{\infty}\left(\frac{\pi_{G g}^{t}(\tilde{\mu})(h-\ell)+\ell(1-h)}{h}\right) \delta^{t} \ell^{t} \\
& <p_{G}(\tilde{\mu}, \hat{y})-\ell+\sum_{t=1}^{\infty}\left(\frac{(h-\ell)+\ell(1-h)}{h}\right) \delta^{t} \ell^{t} \\
& =p_{G}(\tilde{\mu}, \hat{y})-\ell+(1-\ell) \frac{\delta \ell}{1-\delta \ell} \\
& =p_{G}(\tilde{\mu}, \hat{y})-\frac{(1-\delta) \ell}{1-\delta \ell}<p_{G}(\tilde{\mu}, \hat{y})
\end{aligned}
$$

where the second inequality follows from $\pi_{G g}^{t}(\tilde{\mu})<1$. Thus, we have reached a contradictory conclusion that (36) cannot hold at $\tilde{\mu}$. This completes the proof of (33).

Step 2: We now show that

[A] $p_{G}^{*}(\mu)$ and $V_{\ell}^{*}(\mu)$ are continuous and strictly increasing in $\mu \in\left(\mu^{\prime}-\varepsilon, 1\right]$ for sufficiently small $\varepsilon>0$.

For $\mu>\mu^{\prime}-\varepsilon$, let $y_{\mu}$ denote the unique solution to $\pi_{B b}\left(\mu, y_{\mu}\right)=\mu^{\prime}$ if a solution exists, and let $y_{\mu}=0$ otherwise. For sufficiently small $\varepsilon>0$, (33) and continuity of $y^{*}(\mu)$ imply that $y^{*}(\mu)>y_{\mu}$ for all $\mu>\mu^{\prime}-\varepsilon$.

Consider the graph of $\delta\left(V_{\ell}^{*}\left(\pi_{B b}(\mu, \cdot)\right)-V_{\ell}^{*}(0)\right)$ as a function of $y$, called the "loss graph." Note that $V_{\ell}^{*}(\mu)$ is continuous and strictly increases in $\mu \in\left(\mu^{\prime}, 1\right]$ and $\pi_{B b}(\mu, y)>\mu^{\prime}$ for all $\mu>\mu^{\prime}-\varepsilon$ and $y>y_{\mu}$ by definition of $y_{\mu}$. Thus, for $\mu>\mu^{\prime}-\varepsilon$ and $y>y_{\mu}$, the loss graph

i) is continuous and strictly increases in $y$ with terminal value $\delta\left(V_{\ell}^{*}(1)-V_{\ell}^{*}(0)\right)=\delta \Delta$;

ii) continuously "shifts downward" as $\mu$ decreases.

On the other hand, the graph of $p_{G}(\mu, \cdot)$ as a function of $y \in[0,1]$, which we call the "gain graph,"

iii) is continuous and strictly decreases in $y$ for $y<1$, and

iv) continuously shifts downward as $\mu$ decreases.

In addition, when $\mu=\bar{\mu}$ the two graphs touch at $y=y^{*}(\bar{\mu})=1$.

Consider $\mu \in\left(\mu^{\prime}-\varepsilon, \bar{\mu}\right)$. Given that $y^{*}(\mu)>y_{\mu}$ for $\mu \in\left(\mu^{\prime}-\varepsilon, \bar{\mu}\right]$, which is implied by (33) and continuity of $y^{*}$ at $\mu^{\prime}$ for sufficiently small $\varepsilon>0$, the loss graph and the gain graph cross at a unique point in $\left(y_{\mu}, 1\right]$, which must be equal to $y^{*}(\mu)$ because $y^{*}(\mu)>y_{\mu}$.

Since, in the domain $y>y_{\mu}$, the two graphs shift continuously as $\mu$ changes in $\left(\mu^{\prime}-\right.$ $\varepsilon, \bar{\mu}]$, both this unique intersection point, $y^{*}(\mu)$, and the value of the gain graph at the intersection point, $p_{G}^{*}(\mu)$, change continuously in $\left(\mu^{\prime}-\varepsilon, \bar{\mu}\right]$. Furthermore, since the two graphs strictly shift upward as $\mu$ increases in $\left(\mu^{\prime}-\varepsilon, \bar{\mu}\right]$, it follows that $p_{G}^{*}(\mu)$ strictly increases in $\mu \in\left(\mu^{\prime}-\varepsilon, \bar{\mu}\right]$. Consequently, in conjunction with $y^{*}(\mu)=1$ for $\mu \geq \bar{\mu}$, we have verified that $p_{G}^{*}(\mu)$ is continuous and strictly increasing in $\mu \in\left(\mu^{\prime}-\varepsilon, 1\right]$. Equation (32) ensures that the same property holds for $V_{\ell}^{*}(\mu)$ and thereby, [A] above holds.

Step 3: As the last step of the proof, let $\mu^{\prime} \geq \mu_{0}$ be the infimum of $\mu^{\prime} \in\left(\mu_{0}, \bar{\mu}\right]$ such that $V_{\ell}^{*}(\mu)$ is continuous and strictly increasing in $\mu \in\left(\mu^{\prime}, 1\right]$. If $\underline{\mu}^{\prime}>\mu_{0},(33)$ and $[\mathrm{A}]$ 
would lead to a contradiction to the fact that $\underline{\mu}^{\prime}$ is the infimum of such points, completing the proof of the Lemma.

Lemma $6 y^{*}$ is continuous at all $\mu \in(0,1]$.

Proof. We already showed that it is continuous on $\left[\mu_{0}, 1\right]$ for some $\mu_{0}<\bar{\mu}$ in the preceding proof. To reach a contradiction, suppose $y^{*}$ is discontinuous at some points and let $\mu^{d} \in$ $\left(0, \mu_{0}\right]$ be the supremum of these points. Then $y^{*}$ is continuous at all $\mu>\mu^{d}$. By Lemma $5, \pi_{B b}\left(\mu, y^{*}(\mu)\right)>\mu$ for all $\mu \in\left(\mu^{d}, 1\right)$, and $p_{G}^{*}(\mu)$ and $V_{\ell}^{*}(\mu)$ are continuous and strictly increasing in $\mu \in\left(\mu^{d}, 1\right]$. This means that for $\mu \in\left(\mu^{d}, \bar{\mu}\right)$, the values $y^{*}(\mu)$ and $p_{G}^{*}(\mu)$ are determined by the unique intersection point of the gain graph and the loss graph on the range of $y$ such that $\pi_{B b}(\mu, y) \geq \mu^{d}$.

Moreover, the same argument as in equation (38) shows that for $\mu\left(>\mu^{d}\right)$ close to $\mu^{d}$ :

$$
\begin{aligned}
V_{\ell}^{*}\left(\pi_{B b}\left(\mu, y^{*}(\mu)\right)\right)-V_{\ell}^{*}(0) & <p_{G}\left(\pi_{B b}\left(\mu, y^{*}(\mu)\right), \hat{y}\right)-\frac{(1-\delta) \ell}{1-\delta \ell} \\
\text { and } p_{G}\left(\mu, y^{*}(\mu)\right) & =\delta\left(V_{\ell}^{*}\left(\pi_{B b}\left(\mu, y^{*}(\mu)\right)\right)-V_{\ell}^{*}(0)\right)
\end{aligned}
$$

which yield

$$
p_{G}\left(\mu^{d}, \lim _{\mu \downarrow \mu^{d}} y^{*}(\mu)\right) \leq \delta\left(p_{G}\left(\pi_{B b}\left(\mu^{d}, \lim _{\mu \downarrow \mu^{d}} y^{*}(\mu)\right), \hat{y}\right)-\frac{(1-\delta) \ell}{1-\delta \ell}\right)<p_{G}\left(\pi_{B b}\left(\mu^{d}, \lim _{\mu \downarrow \mu^{d}} y^{*}(\mu)\right), \hat{y}\right) .
$$

These inequalities cannot hold if $\lim _{\mu \downarrow \mu^{d}} y^{*}(\mu)=\hat{y}$, because $\pi_{B b}\left(\mu^{d}, \hat{y}\right)=\mu^{d}$. Hence, $\lim _{\mu \downarrow \mu^{d}} y^{*}(\mu)>\hat{y}$.

If $y^{*}(\mu) \geq \hat{y}+\varepsilon$ in a neighborhood of $\mu^{d}$ for some $\varepsilon>0$, then since $\pi_{B b}\left(\mu, y^{*}(\mu)\right)>\mu^{d}$, the same logic used to prove $[\mathrm{A}]$ would verify that $y^{*}(\cdot)$ is continuous in a neighborhood of $\mu^{d}$, contradicting the definition of $\mu^{d}$. Hence, there exists a sequence $\mu_{n}\left(<\mu^{d}\right)$ converging to $\mu^{d}$ such that $\lim _{n \rightarrow+\infty} y^{*}\left(\mu_{n}\right) \leq \hat{y}<\lim _{\mu \downarrow \mu^{d}} y^{*}(\mu)$.

In particular, $\lim _{n \rightarrow+\infty} p_{G}\left(\mu_{n}, y^{*}\left(\mu_{n}\right)\right)>\lim _{\mu \downarrow \mu^{d}} p_{G}^{*}(\mu)$. Since $y^{*}(\mu)<1$ for $\mu=\mu_{n}$ and $\mu \in\left(\mu^{d}, \bar{\mu}\right)$,

$$
p_{G}\left(\mu, y^{*}(\mu)\right)=\delta\left(V_{\ell}^{*}\left(\pi_{B b}\left(\mu, y^{*}(\mu)\right)\right)-V_{\ell}^{*}(0)\right)
$$

holds for $\mu=\mu_{n}$ and $\mu \in\left(\mu^{d}, \bar{\mu}\right)$. Thus, $\lim _{n \rightarrow+\infty} V_{\ell}^{*}\left(\pi_{B b}\left(\mu_{n}, y^{*}\left(\mu_{n}\right)\right)\right)>\lim _{\mu \downarrow \mu^{d}} V_{\ell}^{*}\left(\pi_{B b}\left(\mu, y^{*}(\mu)\right)\right)$. Given $\lim _{n \rightarrow+\infty} \pi_{B b}\left(\mu_{n}, y^{*}\left(\mu_{n}\right)\right)=\pi_{B b}\left(\mu^{d}, \lim _{n \rightarrow+\infty} y^{*}\left(\mu_{n}\right)\right) \leq \mu^{d}=\pi_{B b}\left(\mu^{d}, \hat{y}\right)$, we find that

$$
\lim _{\mu \downarrow \mu^{d}} V_{\ell}^{*}\left(\pi_{B b}\left(\mu, y^{*}(\mu)\right)\right)<\sup _{\mu \leq \mu^{d}} V_{\ell}^{*}(\mu) .
$$

Take $\mu^{\prime \prime}<\mu^{d}$ such that $V_{\ell}^{*}\left(\mu^{\prime \prime}\right)$ is arbitrarily close to $\sup _{\mu \leq \mu^{d}} V_{\ell}^{*}(\mu)<V_{\ell}^{*}(1) .{ }^{19}$ As (39) implies that $\lim _{\mu \downarrow \mu^{d}} V_{\ell}^{*}(\mu)<\sup _{\mu \leq \mu^{d}} V_{\ell}^{*}(\mu)$, there is a unique $\mu^{+}>\mu^{d}$ such that $V_{\ell}^{*}\left(\mu^{+}\right)=\sup _{\mu \leq \mu^{d}} V_{\ell}^{*}(\mu)$. Then, $\pi_{B b}\left(\mu^{+}, y^{*}\left(\mu^{+}\right)\right)>\mu^{+}$by Lemma 5 and, since $y^{*}\left(\mu^{+}\right)>0$,

$$
p_{G}^{*}\left(\mu^{+}\right) \geq \delta\left(V_{\ell}^{*}\left(\pi_{B b}\left(\mu^{+}, y^{*}\left(\mu^{+}\right)\right)\right)-V_{\ell}^{*}(0)\right) .
$$

\footnotetext{
${ }^{19}$ The last inequality is verified as follows: For $V_{\ell}^{*}(\mu)$ to be arbitrarily close to $V_{\ell}^{*}(1)$ for some $\mu \leq \mu^{d}$, we need $p_{G}^{*}(\mu)$ arbitrarily close to 1 by $(29)$, but then $y^{*}(\mu)=1$ would be optimal due to $\delta \Delta<1$, contradicting $p_{G}^{*}(\mu)$ being arbitrarily close to 1 .
} 
We then verify that $p_{G}^{*}\left(\mu^{+}\right) \geq p_{G}^{*}\left(\mu^{\prime \prime}\right)$. This is immediate if $\pi_{B b}\left(\mu^{+}, y^{*}\left(\mu^{+}\right)\right)=1$ since $p_{G}^{*}\left(\mu^{\prime \prime}\right)<\delta\left(V_{\ell}^{*}(1)-V_{\ell}^{*}(0)\right)$. If $\pi_{B b}\left(\mu^{+}, y^{*}\left(\mu^{+}\right)\right)<1$, the gain and loss graphs for $\mu^{\prime \prime}$ cannot cross at any $y$ such that $V_{\ell}^{*}\left(\pi_{B b}\left(\mu^{\prime \prime}, y\right)\right)>V_{\ell}^{*}\left(\pi_{B b}\left(\mu^{+}, y^{*}\left(\mu^{+}\right)\right)\right.$. To see this, note that the two graphs crossing at such $y$ would require that $\pi_{B b}\left(\mu^{\prime \prime}, y\right)>\pi_{B b}\left(\mu^{+}, y^{*}\left(\mu^{+}\right)\right)$ and thus $y>y^{*}\left(\mu^{+}\right)$, but this would imply that (40) holds with equality and, therefore, $p_{G}\left(\mu^{\prime \prime}, y\right)<p_{G}^{*}\left(\mu^{+}\right)<\delta\left(V_{\ell}^{*}\left(\pi_{B b}\left(\mu^{\prime \prime}, y\right)\right)-V_{\ell}^{*}(0)\right)$, contradicting the two graphs crossing at $y$. Then, $y^{*}\left(\mu^{\prime \prime}\right)<1$ and thus, $p_{G}^{*}\left(\mu^{\prime \prime}\right)=\delta\left(V_{\ell}^{*}\left(\pi_{B b}\left(\mu^{\prime \prime}, y^{*}\left(\mu^{\prime \prime}\right)\right)\right)-V_{\ell}^{*}(0)\right) \leq p_{G}^{*}\left(\mu^{+}\right)$from (40), verifying the claim.

Furthermore, $V_{\ell}^{*}\left(\pi_{G g}^{*}\left(\mu^{+}\right)\right)>V_{\ell}^{*}\left(\pi_{G g}^{*}\left(\mu^{\prime \prime}\right)\right)$ because $\pi_{G g}^{*}\left(\mu^{+}\right)>\max \left\{\mu^{+}, \pi_{G g}^{*}\left(\mu^{\prime \prime}\right)\right\}$ and $V_{\ell}^{*}$ is strictly increasing in $\mu>\mu^{+}$and $V_{\ell}^{*}\left(\mu^{+}\right)=\sup _{\mu \leq \mu^{d}} V_{\ell}^{*}(\mu)$. In light of (29), these observations dictate $V_{\ell}^{*}\left(\mu^{\prime \prime}\right)<V_{\ell}^{*}\left(\mu^{+}\right)$, contradicting to $V_{\ell}^{*}\left(\mu^{\prime \prime}\right)$ being arbitrarily close to $\sup _{\mu \leq \mu^{d}} V_{\ell}^{*}(\mu)=V_{\ell}^{*}\left(\mu^{+}\right)$. This establishes that $y^{*}$ is continuous at all $\mu \in\left(0, \mu_{0}\right]$.

Lemmas 5 and 6 imply that $V_{\ell}^{*}$ is continuous and strictly increasing on $(0,1]$.

To complete the proof of Lemma 2 , consider the possibility that $V_{\ell}^{*}$ is discontinuous at $\mu=0$, i.e., $V_{\ell}^{*}(0)<V_{\ell}^{*}\left(0^{+}\right)=\lim _{\mu \downarrow 0} V_{\ell}^{*}(\mu)$. If $\delta\left(V_{\ell}^{*}\left(0^{+}\right)-V_{\ell}^{*}(0)\right) \leq \ell=p_{G}(0,1)$, then $y^{*}(\mu) \rightarrow 1$ as $\mu \rightarrow 0$ and thus, we would have $V_{\ell}^{*}\left(0^{+}\right)=\ell+\delta\left(\ell V_{\ell}^{*}\left(0^{+}\right)+(1-\ell) V_{\ell}^{*}(0)\right) \Rightarrow$ $V_{\ell}^{*}\left(0^{+}\right)=V_{\ell}^{*}(0)$, a contradiction. If $\delta\left(V_{\ell}^{*}\left(0^{+}\right)-V_{\ell}^{*}(0)\right)>\ell$, then $\lim _{\mu \downarrow 0} p_{G}^{*}(\mu)=\delta\left(V_{\ell}^{*}\left(0^{+}\right)-\right.$ $\left.V_{\ell}^{*}(0)\right)$ and thus, we would have $V_{\ell}^{*}\left(0^{+}\right)=\delta\left(V_{\ell}^{*}\left(0^{+}\right)-V_{\ell}^{*}(0)\right)+\delta\left(\ell V_{\ell}^{*}\left(0^{+}\right)+(1-\ell) V_{\ell}^{*}(0)\right) \Rightarrow$ $V_{\ell}^{*}\left(0^{+}\right)=-\delta \ell V_{\ell}^{*}(0) /(1-\delta-\delta \ell) \Rightarrow V_{\ell}^{*}\left(0^{+}\right)-V_{\ell}^{*}(0)=\frac{\ell}{\delta+\delta \ell-1}>\frac{\ell}{\delta \ell}>1>\Delta$, a contradiction to (26). This completes the proof of Lemma 2.

\section{A.4 Proof of Proposition 1}

\section{A.4.1 Proof of existence of a fixed point of $T$ in $\mathcal{F}$}

Recall that the equilibrium value function $V_{\ell}^{*}$ is a fixed point of an operator defined on $\mathcal{F}$, the set of all continuous and strictly increasing functions on $[0,1]$ with an initial value of $\ell /(1-\delta)$ and an end value of $\ell /(1-\delta)+\Delta$. However, we cannot apply a fixed point theorem to $T$ because $\mathcal{F}$ is not a compact space under a suitable topology. Nor can we extend $T$ to the set of all continuous and bounded functions on $[0,1]$ and apply Blackwell Theorem because $T$ is not non-decreasing.

To solve this problem, we extend $T$ to the set $\overline{\mathcal{F}}$ of all right-continuous and nondecreasing functions $V$ on $[0,1]$ with $V(0)=\ell /(1-\delta)$ and $V(1)=V(0)+\Delta$. To characterize $V_{\ell}^{*}$ as a fixed point of the operator defined on $\overline{\mathcal{F}}$, we extend the definition of the "optimal response" function $y_{V}$ to all function $V \in \overline{\mathcal{F}}$. Note that since $\bar{\mu}$ is independent of $V$, (18) determines $y_{V}(0)=y_{V}(\mu)=1$ for all $\mu \geq \bar{\mu}$. For $0<\mu<\bar{\mu}$, we extend the definition of $y_{V}(\mu)$ to be the unique $y \in(0,1)$ that satisfies

$$
\delta \lim _{y^{\prime} \uparrow y}\left(V\left(\pi_{B b}\left(\mu, y^{\prime}\right)\right)-V(0)\right) \leq p_{G}(\mu, y) \leq \delta\left(V\left(\pi_{B b}(\mu, y)\right)-V(0)\right) .
$$

This uniquely determines the optimal response function $y_{V}$ as

$$
y_{V}(\mu)= \begin{cases}1 & \text { if } \mu=0 \text { or } \mu>\bar{\mu} \\ \text { the unique } y \text { that satisfies (41) } & \text { if } 0<\mu \leq \bar{\mu}\end{cases}
$$


Lemma 7 For any $V \in \overline{\mathcal{F}}, y_{V}(\mu)$ is continuous and strictly positive on $[0,1]$ and $p_{G}\left(\mu, y_{V}(\mu)\right)$ is nondecreasing in $\mu$.

Proof. For each $\mu \in(0, \bar{\mu}], y_{V}(\mu)$ is the value of $y$ at which the gain graph of $p_{G}(\mu, y)$ intersects with the "connected" loss graph of $\delta\left(V\left(\pi_{B b}(\mu, y)\right)-V(0)\right)$, i.e., the latter graph is connected vertically at every discontinuity points by the shortest distance. Since $p_{G}(\mu, 0)=$ $1>\delta \Delta$, the intersection takes place at some $y>0$, establishing that $y_{V}(\mu)>0$ for $\mu \in(0, \bar{\mu}]$ as well as when $\mu=0$ and $\mu>\bar{\mu}$ as per $(42)$.

Let us now prove continuity of $y_{V}$. For each $\mu \in(0, \bar{\mu}]$, denoting $V^{-}\left(\pi_{B b}(\mu, y)\right)=$ $\lim _{x \uparrow y} V\left(\pi_{B b}(\mu, x)\right)$, from (41) we have

$$
\delta\left(V^{-}\left(\pi_{B b}\left(\mu, y_{V}(\mu)\right)\right)-V(0)\right) \leq p_{G}\left(\mu, y_{V}(\mu)\right) \leq \delta\left(V\left(\pi_{B b}\left(\mu, y_{V}(\mu)\right)\right)-V(0)\right) .
$$

Now let $\mu_{n} \rightarrow \mu$ and suppose that $y_{V}\left(\mu_{n}\right) \rightarrow y^{\prime}<y_{V}(\mu)$. Then, as $\pi_{B b}$ increases in $y$, $\pi_{B b}\left(\mu_{n}, y_{V}\left(\mu_{n}\right)\right) \rightarrow \pi_{B b}\left(\mu, y^{\prime}\right)<\pi_{B b}\left(\mu, y_{V}(\mu)\right)$ so that for $n$ large enough we would have

$$
\delta\left(V\left(\pi_{B b}\left(\mu_{n}, y_{V}\left(\mu_{n}\right)\right)\right)-V(0)\right) \leq \delta\left(V^{-}\left(\pi_{B b}\left(\mu, y_{V}(\mu)\right)\right)-V(0)\right)
$$

because $V$ is non-decreasing. But this and the second inequality of (43) imply

$$
\delta\left(V^{-}\left(\pi_{B b}\left(\mu, y_{V}(\mu)\right)\right)-V(0)\right) \geq \lim _{n \rightarrow+\infty} p_{G}\left(\mu_{n}, y_{V}\left(\mu_{n}\right)\right)=p_{G}\left(\mu, y^{\prime}\right)>p_{G}\left(\mu, y_{V}(\mu)\right),
$$

which contradicts the first inequality of (43). As a symmetric reasoning applies for $y^{\prime}>$ $y_{V}(\mu)$, we deduce that $y_{V}\left(\mu_{n}\right) \rightarrow y_{V}(\mu)$.

Furthermore, note that $y_{V}(\mu) \rightarrow 1$ as $\mu \rightarrow 0$ because, for every $y<1, \pi_{B b}(\mu, y) \rightarrow 0$ as $\mu \rightarrow 0$ and thus, $\delta\left(V\left(\pi_{B b}(\mu, y)\right)-V(0)\right)<\ell \leq p_{G}(\mu, y)$ for all $\mu$ sufficiently small. Since $y_{V}(\bar{\mu})=1$ by construction (using $y_{V}(0)=1$ if $\bar{\mu}=0$ ), it follows that $y_{V}(\mu)$ is continuous on $[0,1]$.

For $\mu \geq \bar{\mu}$, we have $p_{G}\left(\mu, y_{V}(\mu)\right)=p_{G}(\mu, 1)$ which increases in $\mu$. For $\mu \in(0, \bar{\mu})$, the two aforementioned, gain and connected loss graphs move upward as $\mu$ increases because both $p_{G}(\mu, y)$ and $\pi_{B b}(\mu, y)$ increases in $\mu$ by (4) and (5), respectively. Hence, the height of the intersection point also increases, i.e., $p_{G}\left(\mu, y_{V}(\mu)\right)$ weakly increases in $\mu$.

Since $\pi_{G g}^{*}(\mu)$ increases in $\mu, T(V)$ as defined in (19) is non-decreasing and rightcontinuous in $\mu$ by Lemma 7 . In addition, $T(V)(0)=p_{G}(0,1)+\delta(\ell V(0)+(1-\ell) V(0))$ which yields $T(V)(0)=\ell /(1-\delta)$; and $T(V)(1)=1+\delta(\ell V(1)+(1-\ell) V(0))$ which yields $T(V)(1)=\ell /(1-\delta)+\Delta$. Hence, the operator $T$ is well-defined on $\overline{\mathcal{F}}$ by (19). We now show that $T$ has a fixed point in $\overline{\mathcal{F}}$, then show that it has to be in $\mathcal{F}$.

A fixed point exists in $\overline{\mathcal{F}}$. Endowed with the topology of the weak convergence, the set $\overline{\mathcal{F}}$ is convex and compact (Theorem 5.1, Billingsley, 1999). By Fan-Glicksberg Fixed Point Theorem, ${ }^{20}$ therefore, $T$ has a fixed point in $\overline{\mathcal{F}}$ if $T$ is continuous on $\overline{\mathcal{F}}$, which we show below.

\footnotetext{
${ }^{20}$ This theorem (Fan, 1952; Glicksberg, 1952) states that an upper hemi-continuous convex valued correspondence from a nonempty compact convex subset of a convex Hausdorff topological vector space has a fixed point.
} 
Consider a sequence $V_{n}, n=1,2, \cdots$, in $\overline{\mathcal{F}}$ that weakly converges to $V$. To prove continuity of $T$, we show below that $T\left(V_{n}\right)$ weakly converges to $T(V)$, i.e., $T\left(V_{n}\right)(\mu)$ converges to $T(V)(\mu)$ at all continuity points of $T(V)$ (Theorem 2.1, Billingsley, 1999).

Let $\Omega$ be the set of all points where $V\left(\pi_{G g}^{*}(\mu)\right)$ is continuous. As $\pi_{G g}^{*}(\mu)$ is increasing, $[0,1] \backslash \Omega$ is countable. As $V$ is continuous at $\pi_{G g}^{*}(\mu)$ if $\mu \in \Omega$, weak convergence of $V_{n}$ implies that $V_{n}\left(\pi_{G g}^{*}(\mu)\right)$ converges to $V\left(\pi_{G g}^{*}(\mu)\right)$ on $\Omega$.

Next, let $y_{V}(\mu)$ be as defined in (42) for $V$ and $y_{V_{n}}(\mu)$ for $V_{n}$. We now show that $y_{V_{n}}(\mu) \rightarrow y_{V}(\mu)$ for all $\mu$.

That $y_{V_{n}}(\mu) \rightarrow y_{V}(\mu)$ is trivial from (42) if $\mu=0$ or $\mu>\bar{\mu}$. Hence, suppose $0<\mu \leq \bar{\mu}$ so that, denoting $V_{n}^{-}\left(\pi_{B b}(\mu, y)\right)=\lim _{x \uparrow y} V_{n}\left(\pi_{B b}(\mu, x)\right)$, we have

$$
\delta\left(V_{n}^{-}\left(\pi_{B b}\left(\mu, y_{V_{n}}(\mu)\right)\right)-V(0)\right) \leq p_{G}\left(\mu, y_{V_{n}}(\mu)\right) \leq \delta\left(V_{n}\left(\pi_{B b}\left(\mu, y_{V_{n}}(\mu)\right)\right)-V(0)\right) .
$$

By taking a subsequence if necessary, we may assume that $y_{V_{n}}(\mu)$ converges to a limit $y^{\prime}$. To reach a contradiction, suppose $y^{\prime} \neq y_{V}(\mu)$. Consider the case that $y^{\prime}<y_{V}(\mu)$. Then, since $p_{G}(\mu, y)$ decreases with $y$ there exists $\varepsilon>0$ such that

$$
p_{G}\left(\mu, y_{V_{n}}(\mu)\right)>p_{G}\left(\mu, y_{V}(\mu)\right)+\varepsilon \geq \delta\left(V^{-}\left(\pi_{B b}\left(\mu, y_{V}(\mu)\right)\right)-V(0)\right)+\varepsilon
$$

for sufficiently large $n$ where the second inequality follows from (43).

Because $V$ is non-decreasing in $\mu$ and $\pi_{B b}(\mu, y)$ is increasing in $y$, there exists some $y^{\prime \prime}$, with $y^{\prime}<y^{\prime \prime}<y_{V}(\mu)$, such that $V(\mu)$ is continuous at $\mu=\pi_{B b}\left(\mu, y^{\prime \prime}\right)$. Then, weak convergence implies that $V_{n}\left(\pi_{B b}\left(\mu, y^{\prime \prime}\right)\right)$ converge to $V\left(\pi_{B b}\left(\mu, y^{\prime \prime}\right)\right)$. Thus, for $n$ large enough,

$\delta V_{n}\left(\pi_{B b}\left(\mu, y_{V_{n}}(\mu)\right)\right) \leq \delta V_{n}\left(\pi_{B b}\left(\mu, y^{\prime \prime}\right)\right)<\delta V\left(\pi_{B b}\left(\mu, y^{\prime \prime}\right)\right)+\varepsilon / 2 \leq \delta V^{-}\left(\pi_{B b}\left(\mu, y_{V}(\mu)\right)\right)+\varepsilon / 2$

where the first inequality follows because $V_{n}\left(\pi_{B b}(\mu, y)\right)$ is non-decreasing in $y$ and the second from convergence of $V_{n}\left(\pi_{B b}\left(\mu, y^{\prime \prime}\right)\right)$. But then the conditions (45) and (46) imply that

$$
p_{G}\left(\mu, y_{V_{n}}(\mu)\right)>\delta\left(V_{n}\left(\pi_{B b}\left(\mu, y_{V_{n}}(\mu)\right)\right)-V(0)\right)+\varepsilon / 2
$$

for sufficiently large $n$, which contradicts (44).

For the case that $y^{\prime}>y_{V}(\mu)$, we can apply the same reasoning using $V_{n}^{-}\left(\pi_{B b}\left(\mu, y_{V_{n}}(\mu)\right)\right)>$ $V_{n}\left(\pi_{B b}\left(\mu, y_{V}(\mu)\right)\right)$ for $n$ large to reach an analogous contradiction:

$$
p_{G}\left(\mu, y_{V_{n}}(\mu)\right)<\delta\left(V_{n}^{-}\left(\pi_{B b}\left(\mu, y_{V_{n}}(\mu)\right)\right)-V(0)\right)-\varepsilon / 2 .
$$

Hence, we conclude that $y_{V_{n}}(\mu) \rightarrow y_{V}(\mu)$ for all $\mu$.

Together with the earlier result that $V_{n}\left(\pi_{G g}^{*}(\mu)\right) \rightarrow V\left(\pi_{G g}^{*}(\mu)\right)$ for all $\mu \in \Omega$, this establishes for all $\mu \in \Omega$ that

$$
\begin{aligned}
T\left(V_{n}\right)(\mu) & =p_{G}\left(\mu, y_{V_{n}}(\mu)\right)+\delta\left(\ell V_{n}\left(\pi_{G g}^{*}(\mu)\right)+(1-\ell) V(0)\right) \\
& \rightarrow p_{G}\left(\mu, y_{V}(\mu)\right)+\delta\left(\ell V\left(\pi_{G g}^{*}(\mu)\right)+(1-\ell) V(0)\right)=T(V)(\mu)
\end{aligned}
$$

as $n \rightarrow \infty$. Finally, to verify this convergence at every continuity point of $T(V)(\mu)$, observe first that this convergence is trivial from (42) at $\mu=0,1$. For any other $\mu \notin \Omega$ at which 
$T(V)$ is continuous, one can find $\mu_{1} \in \Omega \cap(0, \mu)$ arbitrarily close to $\mu$ and $\mu_{2} \in \Omega \cap(\mu, 1)$ arbitrarily close to $\mu$ because $\Omega$ is dense in $[0,1]$. Since $T\left(V_{n}\right)\left(\mu_{1}\right) \leq T\left(V_{n}\right)(\mu) \leq T\left(V_{n}\right)\left(\mu_{2}\right)$ and $T(V)\left(\mu_{1}\right) \leq T(V)(\mu) \leq T(V)\left(\mu_{2}\right)$, taking the limits we get

$$
\begin{gathered}
T(V)\left(\mu_{1}\right) \leq \liminf T\left(V_{n}\right)(\mu) \leq \lim \sup T\left(V_{n}\right)(\mu) \leq T(V)\left(\mu_{2}\right), \quad \text { and } \\
\sup _{\substack{\mu_{1} \in \Omega \\
\mu_{1}<\mu}} T(V)\left(\mu_{1}\right)=T(V)(\mu)=\inf _{\substack{\mu_{2} \in \Omega \\
\mu_{2}>\mu}} T(V)\left(\mu_{2}\right),
\end{gathered}
$$

which imply, as desired, that $T\left(V_{n}\right)(\mu)$ converges to $T(V)(\mu)$ at every continuity point of $T(V)(\mu)$. This proves that $T$ is continuous and thus, completes the proof of existence of a fixed point $V_{\ell}^{*}$ in $\overline{\mathcal{F}}$.

A fixed point exists in $\mathcal{F}$. It remains to verify that a fixed point of $T$ in $\overline{\mathcal{F}}$ is indeed in $\mathcal{F}$. For this, just note that the proof of Lemma 2 relies only on equation (29) and the optimality condition (6) applied to the equilibrium value function. Both are verified by any fixed point of $T$ defined on $\overline{\mathcal{F}}$. By Lemma 2, therefore, it has to be in $\mathcal{F}$.

\section{A.4.2 Proof of uniqueness of a fixed point of $T$ in $\mathcal{F}$}

To reach a contradiction, suppose there are two fixed points $V^{1}$ and $V^{2}$ in $\mathcal{F}$. Notice that

$$
V^{i}(\mu)=p_{G}(\mu, 1)+\delta(\ell V(1)+(1-\ell) V(0)) \quad \forall \mu \geq \bar{\mu}, \quad i=1,2,
$$

in particular, $V^{1}(\mu)=V^{2}(\mu)$ for all $\mu \geq \bar{\mu}$. Thus, the following is well-defined:

$$
\hat{\mu}:=\min \left\{\mu \mid V^{1}\left(\mu^{\prime}\right)=V^{2}\left(\mu^{\prime}\right) \forall \mu^{\prime} \geq \mu\right\} \in(0, \bar{\mu}] .
$$

A "segment" for $i=1,2$, is a nonempty interval $I_{i}=[x, z] \subset[0, \bar{\mu}]$ such that $V^{i}(\mu)>V^{j}(\mu)$ for all $\mu \in(x, z)$ and $V^{i}(\mu)=V^{j}(\mu)$ for $\mu=x, z$, where $j \neq i$. A "region" for $i=1,2$, is a nonempty interval $R_{i}=[x, z] \subset[0, \bar{\mu}]$ such that $V^{i}(\mu) \geq V^{j}(\mu)$ for all $\mu \in I_{i}$ and there are $x^{\prime}, z^{\prime} \in R_{i}$ such that $\left[x, x^{\prime}\right]$ and $\left[z^{\prime}, z\right]$ are segments for $i$. Let

$$
p_{G}^{i}(\mu):=p_{G}\left(\mu, y_{V^{i}}(\mu)\right) \quad \text { and } \quad \pi_{B b}^{i}(\mu):=\pi_{B b}\left(\mu, y_{V^{i}}(\mu)\right) \quad \text { for } \quad i=1,2 \text {. }
$$

Recall that in the proof of Lemma 7 , we have shown that both $p_{G}^{i}(\mu)$ and $\pi_{B b}^{i}(\mu)$ weakly increase in $\mu$. Since $V^{i}$ strictly increases in $\mu$ by Lemma 2 , the same reasoning used in the proof of Lemma 7 establishes that

[B] $p_{G}^{i}(\mu)$ and $\pi_{B b}^{i}(\mu)$ strictly increase in $\mu$.

Next, we establish the following:

[C] If $V^{1}\left(\pi_{B b}^{i}(\mu)\right)=V^{2}\left(\pi_{B b}^{i}(\mu)\right)$ for some $\mu>0$ and some $i=1,2$, then $y_{V^{1}}(\mu)=y_{V^{2}}(\mu)$ and consequently, $p_{G}^{1}(\mu)=p_{G}^{2}(\mu)$ and $\pi_{B b}^{1}(\mu)=\pi_{B b}^{2}(\mu)$. If, in addition, $V^{1}\left(\pi_{G g}^{*}(\mu)\right)=$ $V^{2}\left(\pi_{G g}^{*}(\mu)\right)$ holds, then $V^{1}(\mu)=V^{2}(\mu)$. 
Note that this observation is trivial for $\mu \geq \bar{\mu}$. Since

$$
p_{G}^{i}(\mu)=\delta\left(V^{i}\left(\pi_{B b}^{i}(\mu)\right)-V^{i}(0)\right) \quad \forall \mu \in(0, \bar{\mu}]
$$

$V^{1}\left(\pi_{B b}^{i}(\mu)\right)=V^{2}\left(\pi_{B b}^{i}(\mu)\right)$ implies $p_{G}^{1}(\mu)=p_{G}^{2}(\mu)$, which in turn implies $y_{V^{1}}(\mu)=y_{V^{2}}(\mu)$, from which the remaining claims of $[\mathrm{C}]$ follow.

Finally, since $\pi_{B b}^{i}(\hat{\mu})>\hat{\mu}$ by Lemma 5 and $\pi_{G g}^{*}(\hat{\mu})>\hat{\mu}$ by (12), due to continuity, there is $\mu^{\prime}<\hat{\mu}$ such that $V^{1}\left(\mu^{\prime}\right) \neq V^{2}\left(\mu^{\prime}\right), \pi_{B b}^{i}\left(\mu^{\prime}\right)>\hat{\mu}$ and $\pi_{G g}^{*}\left(\mu^{\prime}\right)>\hat{\mu}$. Then, $V^{1}\left(\pi_{B b}^{i}\left(\mu^{\prime}\right)\right)=$ $V^{2}\left(\pi_{B b}^{i}\left(\mu^{\prime}\right)\right)$ by $(48)$ and thus, $V^{1}\left(\mu^{\prime}\right)=V^{2}\left(\mu^{\prime}\right)$ by [C], a contradiction to the earlier assertion that $V^{1}\left(\mu^{\prime}\right) \neq V^{2}\left(\mu^{\prime}\right)$. This completes the proof of uniqueness, hence the proof of Proposition 1.

\section{A.5 Proof of Proposition 2}

Let $V_{h}(\mu)$ be the value function from the following strategy of an $h$-type seller: always report $q=g$ truthfully and upon drawing $q=b$ for the first time report $m=G$ and get $V_{h}^{*}(0)$ in the continuation subgame. Then,

$$
V_{h}(\mu)=\left[\sum_{t=0}^{\infty} h^{t} \delta^{t} p_{G}\left(\pi_{G g}^{t}(\mu), y^{*}\left(\pi_{G g}^{t}(\mu)\right)\right)\right]+\delta V_{h}^{*}(0)(1-h) \sum_{t=1}^{\infty} h^{t} \delta^{t}
$$

where $\pi_{G g}^{t}(\mu)$ is as defined in (30). Since $V_{h}^{*}(\mu) \geq V_{h}(\mu)$ is clear from definition of $V_{h}^{*}$, it suffices to show $V_{h}(\mu)-V_{\ell}^{*}(\mu)>0$. Subtracting (31) from (51),

$$
V_{h}(\mu)-V_{\ell}^{*}(\mu)=\left[\sum_{t=0}^{\infty}\left(h^{t}-\ell^{t}\right) \delta^{t} p_{G}\left(\pi_{G g}^{t}(\mu), y^{*}\left(\pi_{G g}^{t}(\mu)\right)\right)\right]+\delta\left(\frac{1-h}{1-\delta h}-\frac{1-\ell}{1-\delta \ell}\right) V_{\ell}^{*}(0) .
$$

Since $p_{G}\left(\pi_{G g}^{t}(\mu), y^{*}\left(\pi_{G g}^{t}(\mu)\right)\right)>\ell$ for $\mu>0$, it follows that

$$
V_{h}(\mu)-V_{\ell}^{*}(\mu)>\frac{\delta(h-\ell) \ell}{(1-\delta h)(1-\delta \ell)}-\frac{\delta(1-\delta)(h-\ell)}{(1-\delta h)(1-\delta \ell)} V_{\ell}^{*}(0)=0 .
$$

As $V_{h}^{*}(\mu) \geq V_{h}(\mu)$ and $V_{h}^{*}(0)=V_{\ell}^{*}(0)$, this completes the proof.

\section{A.6 Proof of Proposition 3}

As asserted earlier, an honest equilibrium exists if and only if truth-telling is optimal for an $h$-type seller when $\mu>0$, under the presumption that the market reacts according to $p^{*}$ and $\pi^{*}$ specified in (11) and (12).

By the sorting condition verified by Proposition 2, an $h$-type seller has more incentive to be truthful than an $\ell$-type seller for all $\mu$. Because the short-term gain from lying when quality is bad, $p_{G}^{*}(\mu)$, is the same for both types, an $h$-type seller must find it optimal to truthfully announce a bad quality whenever an $\ell$-type seller is indifferent between lying and not, which is the case when $\mu \in(0, \bar{\mu})$. By the same token, whenever an $\ell$-type seller prefers to tell the truth, which is the case when quality is good, so does an $h$-type seller. 
It remains to consider the case that $\mu \geq \bar{\mu}$ and quality is bad. Then, telling the truth reveals high ability and an $h$-type seller would find it optimal to do so if the long-term loss from lying, $\delta\left(V_{h}^{*}(1)-V_{h}^{*}(0)\right)=\delta(h-\ell) /(1-\delta)$, exceeds the short-term gain, $p_{G}^{*}(\mu)$. As $p_{G}^{*}(\mu)$ reaches a maximum value of 1 at $\mu=1$, the condition $\delta(h-\ell) /(1-\delta) \geq p_{G}^{*}(1)=$ $1 \Leftrightarrow \delta \geq 1 /(h-\ell+1)=\delta_{h}$ ensures that telling the truth is optimal for an $h$-type seller when quality is bad for all $\mu \geq \bar{\mu}$. Thus we have verified that truth-telling is optimal for an $h$-type seller for all $\mu>0$ if and only if $\delta \geq \delta_{h}$, as desired.

\section{B Appendix to Section 5}

Proof of Proposition 4. As $\delta \Delta$ increases in $\delta$ and $p_{G}(\mu, 1)$ increases in $\mu$, (8) implies that $\bar{\mu}(\delta)$ strictly increases in $\delta>\delta_{\ell}$. Consider $\delta_{\ell}<\delta<\delta^{\prime}<1$ and let $y^{*}(\cdot \mid \delta)$ and $y^{*}\left(\cdot \mid \delta^{\prime}\right)$ denote $y^{*}(\cdot)$ for different $\delta$ and similarly for other equilibrium variables. To reach a contradiction, suppose that $y^{*}(\mu \mid \delta) \leq y^{*}\left(\mu \mid \delta^{\prime}\right)$ for some $\mu \in(0, \bar{\mu})$ where $\bar{\mu}=\bar{\mu}(\delta)$. As $y^{*}(\bar{\mu} \mid \delta)=1>y^{*}\left(\bar{\mu} \mid \delta^{\prime}\right), \mu^{\prime}=\max \left\{\mu<\bar{\mu} \mid y^{*}(\mu \mid \delta) \leq y^{*}\left(\mu \mid \delta^{\prime}\right)\right\}$ is well-defined. Note that $y^{\prime}=y^{*}\left(\mu^{\prime} \mid \delta\right)=y^{*}\left(\mu^{\prime} \mid \delta^{\prime}\right)<1$ and thus from optimality condition

$$
p_{G}\left(\mu^{\prime}, y^{\prime}\right)=\delta\left(V_{\ell}^{*}\left(\pi_{B b}\left(\mu^{\prime}, y^{\prime}\right) \mid \delta\right)-V_{\ell}^{*}(0 \mid \delta)\right)=\delta^{\prime}\left(V_{\ell}^{*}\left(\pi_{B b}\left(\mu^{\prime}, y^{\prime}\right) \mid \delta^{\prime}\right)-V_{\ell}^{*}\left(0 \mid \delta^{\prime}\right)\right) .
$$

However, since $y^{*}(\mu \mid \delta) \geq y^{*}\left(\mu \mid \delta^{\prime}\right)$ for all $\mu \geq \mu^{\prime}$, from (32) we have

$$
\begin{gathered}
\delta\left(V_{\ell}^{*}\left(\pi_{B b}\left(\mu^{\prime}, y^{\prime}\right) \mid \delta\right)-V_{\ell}^{*}(0 \mid \delta)\right)=\sum_{t=0}^{\infty} \delta^{t+1} \ell^{t}\left(p_{G}\left(\pi_{G g}^{t}\left(\mu^{\prime}\right), y^{*}\left(\pi_{G g}^{t}\left(\mu^{\prime}\right) \mid \delta\right)\right)-\ell\right) \\
<\sum_{t=0}^{\infty}\left(\delta^{\prime t+1} \ell^{t}\left(p_{G}\left(\pi_{G g}^{t}\left(\mu^{\prime}\right), y^{*}\left(\pi_{G g}^{t}\left(\mu^{\prime}\right) \mid \delta^{\prime}\right)\right)-\ell\right)=\delta^{\prime}\left(V_{\ell}^{*}\left(\pi_{B b}\left(\mu^{\prime}, y^{\prime}\right) \mid \delta^{\prime}\right)-V_{\ell}^{*}\left(0 \mid \delta^{\prime}\right)\right),\right.
\end{gathered}
$$

which contradicts (52). Thus, we conclude that $y^{*}(\mu \mid \delta)>y^{*}\left(\mu \mid \delta^{\prime}\right)$ for all $\mu \in(0, \bar{\mu}(\delta))$. That $y^{*}(\mu \mid \delta)=1>y^{*}\left(\mu \mid \delta^{\prime}\right)$ for all $\mu \in\left[\bar{\mu}(\delta), \bar{\mu}\left(\delta^{\prime}\right)\right)$ is trivial from the definition of $\bar{\mu}(\cdot)$.

Proof of Proposition 5. Note that an $h$-type seller is better off in an honest equilibrium than in the babbling equilibrium if and only if the opposite is true for an $\ell$-type seller. Hence, we examine an $\ell$-type seller in this proof. From $(32)$ and the fact that $y^{*}(\mu)>\hat{y}$ we have :

$$
V_{\ell}^{*}(\mu)-\frac{\ell}{1-\delta}<Y(\mu):=\sum_{t=0}^{\infty} \delta^{t} \ell^{t}\left(p_{G}\left(\pi_{G g}^{t}(\mu), \hat{y}\right)-\ell\right) .
$$

As $\frac{\partial}{\partial \mu} p_{G}(\mu, \hat{y})=(h-\ell) / h$, we have

$$
\begin{aligned}
Y^{\prime}(\mu) & =\sum_{t=0}^{\infty} \delta^{t} \ell^{t} \frac{h-\ell}{h} \frac{\partial \pi_{G g}^{t}(\mu)}{\partial \mu}=\sum_{t=0}^{\infty} \delta^{t} \ell^{t} \frac{h-\ell}{h}\left[\mu\left(\frac{h}{\ell}\right)^{t / 2}+(1-\mu)\left(\frac{\ell}{h}\right)^{t / 2}\right]^{-2} \\
& >\left.\sum_{t=0}^{\infty} \delta^{t} \ell^{t} \frac{h-\ell}{h}\left[\mu\left(\frac{h}{\ell}\right)^{t / 2}+(1-\mu)\left(\frac{\ell}{h}\right)^{t / 2}\right]^{-2}\right|_{\mu=1} \\
& =\sum_{t=0}^{\infty} \delta^{t} \ell^{t} \frac{h-\ell}{h}\left(\frac{\ell}{h}\right)^{t}=\frac{h-\ell}{h-\delta \ell^{2}}
\end{aligned}
$$


where the inequality follows because $\mu\left(\frac{h}{\ell}\right)^{t / 2}+(1-\mu)\left(\frac{\ell}{h}\right)^{t / 2}$ increases in $\mu$ for all $t \geq 0$. Hence,

$$
Y(\mu)<Y(1)-\frac{h-\ell}{h-\delta \ell^{2}}(1-\mu)=\frac{1-\ell}{1-\delta \ell}-\frac{h-\ell}{h-\delta \ell^{2}}(1-\mu) .
$$

Letting $\tilde{\mu}_{t}$ denote the period- $t$ posterior (random variable) in the babbling equilibrium starting from an initial reputation $\mu$, the value function of an $\ell$-type seller in the babbling equilibrium is

$$
W_{\ell}(\mu)=\mathbb{E}\left[\sum_{t=0}^{\infty} \delta^{t}\left(\tilde{\mu}_{t} h+\left(1-\tilde{\mu}_{t}\right) \ell\right) \mid \theta=\ell\right]=\frac{\ell}{1-\delta}+(h-\ell) \mathbb{E}\left[\sum_{t=0}^{\infty} \delta^{t} \tilde{\mu}_{t} \mid \theta=\ell\right] .
$$

As

$\mathbb{E}\left(\mu_{t+1} \mid \mu_{t}, \theta=\ell\right)=\ell \pi_{G}\left(\mu_{t}\right)+(1-\ell) \pi_{B}\left(\mu_{t}\right) \geq f \cdot \mu_{t}$ where $f:=\inf _{0<\mu<1} \frac{\ell \pi_{G}(\mu)+(1-\ell) \pi_{B}(\mu)}{\mu}$, from (55) we have

$$
W_{\ell}(\mu)-\frac{\ell}{1-\delta}=(h-\ell) \cdot \mathbb{E}\left(\sum_{t=0}^{\infty} \delta^{t} \tilde{\mu}_{t} \mid \theta=\ell\right)>X(\mu):=(h-\ell) \mu \sum_{t=0}^{\infty} \delta^{t} f^{t}=\frac{(h-\ell) \mu}{1-\delta f} .
$$

As $\frac{\ell \pi_{G}(\mu)+(1-\ell) \pi_{B}(\mu)}{\mu}$ is quasi-convex in $\mu \in(0,1)$, the value of $f$ is routinely calculated as

$$
f=(\sqrt{h \ell}+\sqrt{(1-h)(1-\ell)})^{2}=1-(\sqrt{h(1-\ell)}-\sqrt{\ell(1-h)})^{2} .
$$

From this ${ }^{21}$ we further derive that

$$
0<1-f<h-\ell
$$

In conjunction with (56), therefore, $W_{\ell}(\mu)>V_{\ell}^{*}(\mu)$ as $\delta \rightarrow 1$ if $\mu>\mu^{*}$ where $\mu^{*}$ satisfies

$$
\frac{(h-\ell) \mu^{*}}{1-f}=1-\frac{h-\ell}{h-\ell^{2}}\left(1-\mu^{*}\right) \Longleftrightarrow \mu^{*}=\frac{\ell(1-\ell)(1-f)}{(h-\ell)\left(h-\ell^{2}+f-1\right)} \in(0,1) .
$$

That $\mu^{*} \in(0,1)$ follows because $\frac{(h-\ell) \mu}{1-f}$ is a linear function of $\mu$ with a slope greater than 1 and $1-\frac{h-\ell}{h-\ell^{2}}(1-\mu)$ is an affine function with a positive slope less than 1 .

As $1-f \rightarrow h$ as $\ell \rightarrow 0$, to verify that $\mu^{*} \rightarrow 0$ as $\ell \rightarrow 0$, we need to show that $\frac{\ell}{h-\ell^{2}+f-1} \rightarrow 0$, or equivalently, that $\frac{h+f-1}{\ell} \rightarrow \infty$ as $\ell \rightarrow 0$. After some routine calculation, we have

$$
\frac{h+f-1}{\ell}=1-2 h+2 \sqrt{\frac{h(1-h)(1-\ell)}{\ell}}
$$

which explodes as $\ell \rightarrow 0$.

\footnotetext{
${ }^{21}$ In particular, $1-f=h-\ell+2(\ell(1-h)-\sqrt{h(1-h)} \sqrt{\ell(1-\ell)})$ and $\ell(1-h)<\sqrt{h(1-h)} \sqrt{\ell(1-\ell)}$.
} 
To verify that $\mu^{*} \rightarrow 0$ as $\ell \rightarrow h$, note that the derivative of $(\sqrt{h(1-\ell)}-\sqrt{\ell(1-h)})^{2}$ with respect to $\ell$ is

$$
-\left(\sqrt{\frac{h}{1-\ell}}+\sqrt{\frac{1-h}{\ell}}\right)(\sqrt{h(1-\ell)}-\sqrt{\ell(1-h)})
$$

which vanishes when $\ell=h$. So, $(1-f) /(h-\ell) \rightarrow 0$ as $\ell \rightarrow h$ and thus, $\mu^{*} \rightarrow 0$ as $\ell \rightarrow h$.

Regarding the values of $\mu^{*}$, according to the calculations using Mathematica, for given $h, \mu^{*}$ is concave in $\ell \in(0, h)$; and the maximum value of $\mu^{*}$ increases in $h$ from around 0.12 at $h=.1$, to 0.16 at $h=.5,0.25$ at $h=.8,0.4$ at $h=.95, .6$ at $h=.99$. When the lower bound $X(\mu)$ is improved by calculating the actual values of $\tilde{\mu}_{2}$ and $\tilde{\mu}_{3}$ and then add $(h-\ell) \mathbb{E}\left(\tilde{\mu}_{3}\right) \sum_{t=3}^{\infty} f^{t}$, the maximum value of $\mu^{*}$ is reduced, for example, to around 0.45 at $h=0.99$.

For large $h$, we may refine the bound as follows. We use (56) to obtain

$$
\begin{aligned}
W_{\ell}(\mu)-\frac{\ell}{1-\delta}> & (h-\ell) \sum_{t=0}^{\infty} \delta^{t} \ell^{t} \pi_{G g}^{t}(\mu) \\
& +(h-\ell) \sum_{t=1}^{\infty} \delta^{t} t(1-\ell) \ell^{t-1} \frac{\mu(1-h) h^{t-1}}{\mu(1-h) h^{t-1}+(1-\mu)(1-\ell) \ell^{t-1}}
\end{aligned}
$$

where the RHS is the payoffs from the events with at most one bad quality draw.

On the other hand, we have :

$$
\begin{aligned}
Y(\mu) & =\sum_{t=0}^{\infty} \delta^{t} \ell^{t}\left(\pi_{G g}^{t}(\mu) \frac{h-\ell}{h}+\ell \frac{1-h}{h}\right) \\
& =(h-\ell) \sum_{t=0}^{\infty} \delta^{t} \ell^{t} \pi_{G g}^{t}(\mu)+(h-\ell)(1-h)\left[\sum_{t=0}^{\infty} \delta^{t} \ell^{t}\left(\frac{\mu h^{t-1}}{\mu h^{t}+(1-\mu) \ell^{t}}+\frac{\ell}{h(h-\ell)}\right)\right]
\end{aligned}
$$

where we use $\pi_{G g}^{t}(\mu)=\frac{\mu h^{t}}{\mu h^{t}+(1-\mu) \ell^{t}}$.

So we have for any $\mu>0, \lim _{\delta \rightarrow 1} W_{\ell}(\mu)>\lim _{\delta \rightarrow 1} V_{\ell}^{*}(\mu)$ when

$$
\sum_{t=1}^{\infty} t(1-\ell) \ell^{t-1} \frac{\mu h^{t-1}}{\mu(1-h) h^{t-1}+(1-\mu)(1-\ell) \ell^{t-1}}>\sum_{t=0}^{\infty} \ell^{t}\left(\frac{\mu h^{t-1}}{\mu h^{t}+(1-\mu) \ell^{t}}+\frac{\ell}{h(h-\ell)}\right)
$$

which holds for large $h$ because the LHS goes to infinity when $h$ tends to 1 while the RHS is bounded by $\frac{1}{(1-\ell)(h-\ell)}$. Notice that if the LHS exceeds this bound for some $\mu$, so it does for all higher $\mu$. Hence, $W_{\ell}(\mu)>V_{\ell}^{*}(\mu)$ for all $\mu$ above a threshold that vanishes as $h \rightarrow 1$ and $\delta \rightarrow 1$. 
Acknowledgements. This research was supported by the Leverhulme Trust and the National Research Foundation of Korea, and was initiated while B. Jullien was visiting the University of Bristol. We are grateful to Heski Bar-Isaac, Luis Cabral, Russel Cooper, Jacques Crémer, YukFai Fong, Christian Hellwig, David Levine, Lucas Maestri, Thomas Mariotti, Jérome Mathis, Andrew McLennan, Fabien Postel-Vinay, Jérôme Renault, Larry Samuelson, Ron Siegel, Joel Sobel, Peter Sorensen, Mike Whinston, as well as four anonymous referees and an editor for useful discussion and comments. We also thank seminar participants at Columbia University, the European University Institute, the Paris School of Economics, Rutgers University, the Universities of Cambridge, Melbourne, Naples, and Oxford, Yonsei University, and the CSIO/IDEI Workshop in Toulouse School of Economics. Any remaining errors are our own. Corresponding author: In-Uck Park, i.park@bristol.ac.uk.

\section{References}

Atkeson, A., C. Hellwig, and G. Ordonez (2012), "Optimal regulation in the presence of reputation concerns," NBER Working Paper 17898.

Bar-Isaac, H. (2003), "Reputation and Survival: learning in a dynamic signalling model", Review of Economic Studies, 70, 231-251.

Bar-Isaac H. and S. Tadelis, (2008), "Seller Reputation," Foundations and Trends in Microeconomics, 4, 273-351

Benabou, R. and G. Laroque (1992), "Using Privileged Information to Manipulate Markets: Insiders, Gurus and Credibility," Quarterly Journal of Economics, 107, 921-958.

Billingsley, P. (1999), Convergence of Probability Measures, 2nd ed., John Wiley \& Sons, Inc.

Board, S. and M. Meyer-Ter-Vehn (2013), "Reputation for Quality," Econometrica, 81, $2381-2462$

Cabral, L., and A. Hortacsu, (2010), "The Dynamics of Seller Reputation: Evidence from eBay," The Journal of Industrial Economics, 58, 54-78.

Canals-Cerda, J. (2008), "The Value of a Good Reputation Online: An Application to Art Auctions," mimeo, Federal Reserve Bank of Philadelphia

Cripps, M., Mailath, G., and L. Samuelson (2004), "Imperfect Monitoring and Impermanent Reputations," Econometrica, 72, 407-432.

Dellarocas, C. (2003). "The Digitization of Word of Mouth: Promise and Challenges of Online Feedback Mechanisms," Management Science, 49, 1407-1424.

Dellarocas, C. (2006), "Reputation Mechanisms", Handbook on Economics and Information Systems (T. Hendershott, ed.), Elsevier Publishing.

Fan, K. (1952), "Fixed point and minimax theorems in locally convex topological linear spaces," Proc. Nat. Acad. Sci. U.S.A., 38, 121-126.

Friedman E., and P. Resnick (2001), "The Social Cost of Cheap Pseudonyms," Journal of Economics and Management Science, 10, 173-199

Gentzkow M. and J.M. Shapiro (2006), "Media Boas and Reputation", Journal of Political 
Economy, 114(2): 280-316.

Glicksberg, I. L. (1952), "A further generalization of the Kakutani fixed point theorem, with application to Nash equilibrium points," Proc. Amer. Math. Soc., 3, 170-174.

Grossman S. (1981), "The Informational Role of Warranties and Private Disclosure about Product Quality," Journal of Law and Economics, 24, 461-483.

Inderst R. and M. Ottaviani (2013), "Sales Talk, Cancellation Terms, and the Role of Consumers Protection," Review of Economic Studies, 80, 1002-1026.

Inderst R. and M. Ottaviani (2012), "Competition through Commissions and Kickbacks," American Economic Review, 102(2): 780-809.

Jin, G. and A. Kato (2006), "Price, Quality and Reputation: Evidence from an Online Field Experiment," RAND Journal of Economics, 37, 983-1005.

Jolivet, G., B. Jullien, and F. Postel-Vinay (2013), "Reputation and Prices on the eMarket: Evidence from a Major French Platform", IDEI Working Paper 13-418

Kreps, D. and R. Wilson (1982), "Reputation and Imperfect Information," Journal of Economic Theory, 27, 245-252.

Mailath, G. and L. Samuelson (2001), "Who Wants a Good Reputation," Review of Economic Studies, 68, 415-441.

Mailath, G. and L. Samuelson (2006), Repeated Games and Reputations: Long-Run Relationships, Oxford University Press.

Milgrom, P. and D.J. Roberts (1982), "Predation, Reputation and Entry Deterrence," Journal of Economic Theory, 27, 280-312.

Morris, S. (2001), "Political Correctness," Journal of Political Economy, 109, 231-265.

Ottaviani, M. and P. Sorensen (2001), "Information aggregation in debate: who should speak first", Journal of Public Economics, 81, 393-42.

Ottaviani, M. and P. Sorensen (2006a) "Professional advice", Journal of Economic Theory, $126,120-142$.

Ottaviani, M. and P. Sorensen (2006b), "Reputational cheap talk," RAND Journal of Economics, 37, 155-175.

Phelan C. (2006), "Public trust and governement betrayal", Journal of Economic Theory, 130: $27-43$.

Seidmann D.J. (1990), "Effective Cheap Talk with Confilicting Interests," Journal of Economic Theory, 50: 445-548.

Shulman J., Coughlan A. and R. Savaskan (2011): "Managing Consumer Return in a Competitive Environment," Management Science, 57, 347-362.

Sobel, J. (1985), "A Theory of Credibility," Review of Economic Studies, 52, 557-573.

Tadelis, S. (1999), "What's in a Name? Reputation as a Tradeable Asset," American Economic Review, 89, 548-563. 